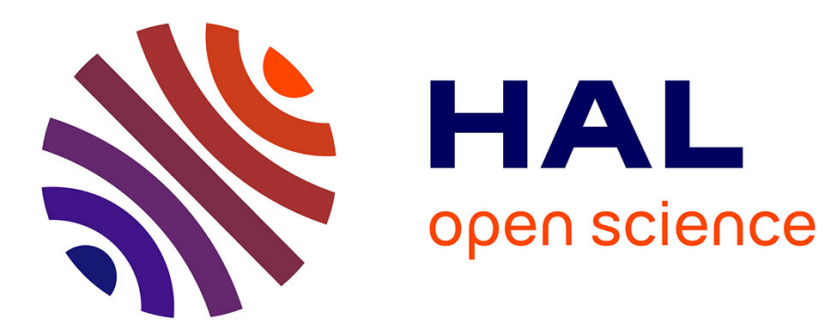

\title{
Wall effects in non-Boussinesq density currents
}

Thomas Bonometti, S. Balachandar, Jacques Magnaudet

\section{To cite this version:}

Thomas Bonometti, S. Balachandar, Jacques Magnaudet. Wall effects in non-Boussinesq density currents. Journal of Fluid Mechanics, 2008, vol. 616, pp. 445-475. 10.1017/S002211200800414X . hal-00862825

\section{HAL Id: hal-00862825 \\ https://hal.science/hal-00862825}

Submitted on 17 Sep 2013

HAL is a multi-disciplinary open access archive for the deposit and dissemination of scientific research documents, whether they are published or not. The documents may come from teaching and research institutions in France or abroad, or from public or private research centers.
L'archive ouverte pluridisciplinaire HAL, est destinée au dépôt et à la diffusion de documents scientifiques de niveau recherche, publiés ou non, émanant des établissements d'enseignement et de recherche français ou étrangers, des laboratoires publics ou privés. 


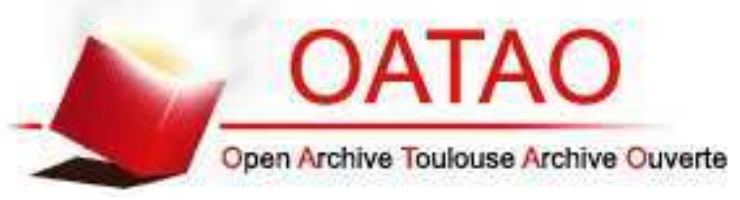

\section{Open Archive TOULOUSE Archive Ouverte (OATAO)}

OATAO is an open access repository that collects the work of Toulouse researchers and makes it freely available over the web where possible.

This is an author-deposited version published in : http://oatao.univ-toulouse.fr/ Eprints ID : 9408

To link to this article : DOI:10.1017/S002211200800414X

URL : http://dx.doi.org/10.1017/S002211200800414X

To cite this version : Bonometti, Thomas and Balachandar, S. and Magnaudet, Jacques. Wall effects in non-Boussinesq density currents.(2008). Journal of Fluid Mechanics, vol. 616 . pp. 445-475. ISSN 0022-1120

Any correspondance concerning this service should be sent to the repository administrator: staff-oatao@ listes-diff.inp-toulouse.fr 


\title{
Wall effects in non-Boussinesq density currents
}

\author{
THOMAS BONOMETTI ${ }^{1}, \dagger$ S. BALACHANDAR ${ }^{1}$ \\ AND JACQUES MAGNAUDET ${ }^{2}$ \\ ${ }^{1}$ Department of Mechanical and Aerospace Engineering, University of Florida, \\ Gainesville, FL 32611, USA \\ ${ }^{2}$ Institut de Mécanique des Fluides de Toulouse, UMR CNRS/INPT/UPS 5502, \\ Allée Camille Soula, 31400 Toulouse, France \\ thomas.bonometti@imft.fr; bala1s@ufl.edu; magnau@imft.fr
}

We report on the results of a numerical study of nearly immiscible contrasted density currents aimed at shedding light on the influence of wall effects on current dynamics in the lock-exchange configuration. The numerical approach is an interface-capturing method which does not involve any explicit reconstruction of the interface. NavierStokes equations are solved on a fixed grid and a hyperbolic equation is used for the transport of the local volume fraction of one of the fluids. This allows us to describe the density currents for the complete range of density contrast $10^{-3} \leqslant \rho_{L} / \rho_{H} \leqslant 0.99$ ( $\rho_{L}$ and $\rho_{H}$ being the density of the light and heavy fluids) and a wide range of Reynolds number $70 \leqslant \operatorname{Re} \leqslant 5 \times 10^{4}$ (based on the channel height and the viscosity of the heavy fluid). The use of free-slip vs. no-slip boundary conditions enables us to separate the dissipation at the interface from the dissipation at the boundaries. Present results reveal that wall effects play a significant role on the propagation of contrasted density currents, unlike dissipation at the interface. It is first shown that when wall friction can be neglected, theoretical models based on the inviscid shallowwater approximations and Benjamin's steady-state result describe fairly well the light and heavy front velocities of density currents for the complete range of density ratio. However, when wall friction cannot be neglected, the results depart significantly from the prediction of inviscid theories. It is observed that most of the dissipation in highly contrasted currents takes place at the bottom wall and is a maximum at the head of the heavy current. This dissipation is shown to be responsible for the decrease of the front velocity. We propose a simple model based on Benjamin's analysis that includes wall friction. Keeping in mind the simplicity and limitations of the present model, the prediction of the front velocity of both the heavy and light currents is observed to be in good agreement with the numerical results for the complete range of density contrast. This gives further support to the idea that wall effects are the crucial ingredient for accurately predicting the front velocity of highly contrasted density currents.

\section{Introduction}

Horizontal density currents are buoyancy-driven flows which manifest themselves as a current of heavy fluid running below light fluid. After release, the current, initially accelerates and reaches a constant speed of spreading (referred to as the slumping phase, Huppert \& Simpson 1980). This phase lasts until the backward-propagating

$\dagger$ Present address: Institut de Mécanique des Fluides de Toulouse, UMR CNRS/INPT/UPS 5502, Allée Camille Soula, 31400 Toulouse, France. Email address for correspondence: thomas.bonometti@imft.fr 
disturbance reflects off the back wall or symmetry plane and propagates forward to catch up with the front (Rottman \& Simpson 1983). The duration of the slumping phase depends on the Reynolds number and volume of release. After the slumping phase, the current velocity decreases in a self-similar manner at a rate that depends on the dominant effect (inertia, viscosity, surface tension).

The constant spreading rate of the slumping phase depends on two parameters, namely the dimensionless height of release, $h_{0} / H$, where the initial height of the release is $h_{0}$ and the total height of the layer is $H$, and the density ratio $\rho_{L} / \rho_{H}$ between the light $\left(\rho_{L}\right)$ and heavy $\left(\rho_{H}\right)$ fluid. As $h_{0} / H$ and $\rho_{L} / \rho_{H}$ vary from 0 to 1 , the extreme cases correspond to quite different configurations. When $h_{0} / H=1$, the problem reduces to a lock-exchange configuration (e.g. fires in enclosed structures, Grant, Jagger \& Lea 1998), while the case $h_{0} / H \ll 1$ corresponds to a small depth release (e.g. oil spilling on the ocean, Hoult 1972). Similarly, when the density difference between the two fluids is small, that is $\rho_{L} / \rho_{H} \approx 1$, the currents are known as Boussinesq currents (e.g. thunderstorm fronts and oceanic currents, Simpson 1997), whereas highly contrasted currents $\left(\rho_{L} / \rho_{H} \approx 0\right)$ are referred to as dam-break flows or cavity flows (e.g. break of a dam, snow avalanches, slug flows in oil pipelines). Clearly, all of these flows are of practical interest since they are encountered in various geophysical, environmental and safety problems. Depending on the specific value of these two parameters, the velocity of the front can vary within a factor of three when spreading over a horizontal plane. However, when spreading down a slope, we must also account for the tilting angle as an extra parameter (Britter \& Linden 1980; Seon, Hulin \& Salin 2004).

Several experiments have studied density currents at the laboratory scale. Their focus has generally been limited to a specific location or region of the parameter space $\left(h_{0} / H ; \rho_{L} / \rho_{H}\right)$ - either Boussinesq flows (Simpson 1972; Huppert \& Simpson 1980; Rottman \& Simpson 1983; Shin, Dalziel \& Linden 2004; Marino, Thomas \& Linden 2005), dam-break flows (Schoklitsch 1917; Martin \& Moyce 1952; Lauber \& Hager 1998; Stansby, Chegini \& Barnes 1998) or cavity flows (Zukoski 1966; Benjamin 1968; Gardner \& Crow 1970; Wilkinson 1982; Baines, Rottman \& Simpson 1985). In these studies, the density contrast was generally kept constant. Indeed, realizing a wide range of density ratios with safe inexpensive fluids of the same nature (gas-gas or liquid-liquid) is difficult. Moreover, for gas-liquid flows, viscous and capillary effects may complicate the experiments if the scale of the apparatus is too small. Therefore, only a few experimental studies have considered varying the density ratio, and experiments with pairs of liquids and pairs of gases are typically limited to density contrasts less than 2 and 20, respectively (Schmidt 1911, Keller \& Chyou 1991, Gröbelbauer, Fanneløp \& Britter 1993; Lowe, Rottman \& Linden 2005).

Recent computational efforts have focused on Boussinesq flows from lockexchange to small release configurations $\left(0<h_{0} / H \leqslant 1 ; \rho_{L} / \rho_{H} \approx 1\right.$, Klemp, Rotunno \& Skemarock 1994; Härtel, Meiburg \& Necker 2000; Ozgökmen et al. 2004; Cantero et al. 2007). These studies made use of spectral approaches and were able to describe accurately the motion of miscible density currents. Computations of moderate to highly contrasted density currents have been rare. The first reported simulation of a non-Boussinesq current is the pioneering work of Daly \& Pracht (1968). By using a marker-in-cell approach, they were able to compute flows of density contrast as high as 3 and show that the Boussinesq approximation was valid for density contrasts of less than 1.2. Outstandingly, some of their simulations also included surface tension effects. Birman, Martin \& Meiburg (2005) extended the application range of spectral methods and considered non-Boussinesq currents with density contrasts up to 5. Independently, Etienne, Hopfinger \& Saramito (2005) used a finite-element 
method with adaptive mesh refinement to compute lock-exchange flows in the range $10^{-2} \leqslant \rho_{L} / \rho_{H}<1$.

The first theoretical attempt to predict the constant spreading rate of density currents was by Ritter (1892) who obtained a solution of the shallow-water equations (de Saint-Venant 1871) in the specific case of a dam break in an infinite ambient fluid $\left(h_{0} / H \approx 0 ; \rho_{L} / \rho_{H} \approx 0\right)$. He found the velocity $u_{H}$ of the heavy front to be (Stoker 1957)

$$
u_{H}=2 \sqrt{g h_{0}} \text {. }
$$

In the other extremity of the parameter space $\left(\rho_{L} / \rho_{H} \approx 1\right)$, Benjamin (1968) reconsidered the work by von Kármán (1940) to obtain the velocities $u_{H}$ and $u_{L}$ of the heavy and light fronts, respectively. Considering the steady-state propagation of a current of density $\rho_{f}$ and height $h$ in a channel filled with fluid of density $\rho_{a}$, and using the balance of volume- and flow-forces he derived an equation for the speed of the current $u_{f}=\left(g\left|\rho_{f}-\rho_{a}\right| h / \rho_{a}\right)^{1 / 2} \mathrm{Fr}(h / H)$ where $\mathrm{Fr}$ is the so-called Benjamin's Froude number function $\operatorname{Fr}(h / H)=\sqrt{(2-h / H)(1-h / H) /(1+h / H)}$. Benjamin also showed that the energy imbalance (called dissipation) is proportional to $(1 / 2-h / H)$. Consequently, a current with $h / H=1 / 2$ is called non-dissipative, and currents with $h / H>1 / 2$ are practically impossible. Assuming the current to be non-dissipative (i.e. $h / H=1 / 2$ ), the heavy and light current velocities can be obtain, namely,

$$
\begin{aligned}
& u_{H}=\frac{1}{2} \sqrt{g \frac{\rho_{H}-\rho_{L}}{\rho_{L}} H}, \\
& u_{L}=\frac{1}{2} \sqrt{g \frac{\rho_{H}-\rho_{L}}{\rho_{H}} H} .
\end{aligned}
$$

This approximation will be referred to as Benjamin's half-depth dissipationless speed result. Note that (1.2) indicate that the speed of the heavy front tends to be $\sqrt{\rho_{H} / \rho_{L}}$ faster than that of the light front.

A theoretical description of non-Boussinesq density currents for the complete range of density ratio in the lock-exchange configuration was provided by Keller \& Chyou (1991) and by Lowe et al. (2005) using a front-matching analysis. This model assumes that the front moves with constant speed and that the light front is half-depth nondissipative and hence its speed is given by $(1.2 b)$ over the entire range of density ratio. The height and speed of the heavy front are then determined by matching the light current with a long wave of expansion connecting either an energy-dissipative heavy current or a hydraulic jump followed by a non-dissipative heavy current, with the aid of the two-layer shallow-water equations (Rottman \& Simpson 1983) and the Benjamin's Froude number function. In particular, in the limit of very high density contrast $\left(\rho_{L} / \rho_{H} \rightarrow 0\right)$, this analysis predicts the velocity of the heavy current to be close to, but slightly smaller than, the value of the front velocity of the dam-break flow, (1.1). Note that the 'dissipation' of these inviscid models is different from the viscous dissipation calculated from the knowledge of the local velocity field, as done in the numerical simulations.

Ungarish (2007) used a one-layer shallow-water theory and obtained the value of the heavy and light front velocities throughout the entire parameter space $\left(h_{0} / H\right.$; $\left.\rho_{L} / \rho_{H}\right)$. Using the fact that the energy in the domain cannot increase, he matched the shallow-water solution of the forward-propagating characteristic with Benjamin's front condition and obtained the height and velocity of the fronts. In the specific case of lock-exchange flows, the predicted velocity of the light front reduces to Benjamin's 
solution $(1.2 b)$, while that of the heavy front is given by

$$
u_{H}=\sqrt{g \frac{\rho_{H}-\rho_{L}}{\rho_{L}} H} \sqrt{\frac{\tilde{h}_{H}\left(2-\tilde{h}_{H}\right)\left(1-\tilde{h}_{H}\right)}{1+\tilde{h}_{H}}},
$$

where $\tilde{h}_{H}=h_{H} / H$ is the dimensionless height of the heavy current. The height of the heavy current is choked to be $\tilde{h}_{H}=1 / 2$ when the density ratio is sufficiently small, i.e. within the range $1 / 8(3-2 \sqrt{2}) \leqslant \rho_{L} / \rho_{H}<1$. For larger density contrast, the height of the heavy current must be obtained by solving

$$
2 \sqrt{\frac{\rho_{L}}{\rho_{H}}}\left(1-\sqrt{\tilde{h}_{H}}\right)=\sqrt{\frac{\tilde{h}_{H}\left(2-\tilde{h}_{H}\right)\left(1-\tilde{h}_{H}\right)}{1+\tilde{h}_{H}}} .
$$

Note that (1.3a) reduces to Ritter's solution (1.1) in the asymptotic case $\rho_{L} / \rho_{H} \rightarrow 0$ and to Benjamin's solution (1.2a) in the case $\rho_{L} / \rho_{H} \rightarrow 1$.

All the above theories were developed by assuming viscous effects both within the fluids and at the boundaries to be negligible. However, an important feature of density currents is that numerous experimental and computational results have reported front velocities lower than all the above theoretical predictions. Although the discrepancy is relatively small at low density contrast (less than $10 \%$, Rottman \& Simpson 1983; Härtel et al. 2000) it becomes significant at moderate density contrast (from $10 \%$ to $30 \%$, Gröbelbauer et al. 1993; Birman et al. 2005; Etienne et al. 2005) and is dramatic at high density contrast (larger than $30 \%$, Schoklitsch 1917; Martin \& Moyce 1952; Dressler 1954; Stansby et al. 1998). Regarding Boussinesq density currents, Klemp et al. (1994) argued that when they take into account friction at the boundaries in their numerical approach, a better agreement with experimental results is observed. Similarly, Huppert \& Simpson (1980) proposed an empirical correction for the front speed of Boussinesq density currents to account better for friction effects. Based on a literature survey of experiments focusing on dam-break waves, Lauber \& Hager (1998) indicate that the effect of boundary roughness becomes significant close to the wave front.

Dressler (1952) and Whitham (1955) were the first to investigate theoretically the effect of resistive forces on shallow flows over rigid horizontal boundaries. By including a drag resistance term modelled by a Chézy closure in the shallow-water equation, they found that the nose of the front was blunt and that the front velocity was reduced. Hogg \& Pritchard (2004) extended the work of Dressler (1952) and Whitham (1955) using matched asymptotic expansions to combine the flow at the front which is affected by resistive forces with the flow in the bulk of the domain within which resistive effects are assumed to be negligible. They also investigated modified dam-break flow where the vertical profile of the longitudinal velocity field was no longer uniform, but included shear. The predicted front velocity was found to be in better agreement with experimental data of dam-break flows. Ermanyuk \& Gavrilov (2007) experimentally investigated the motion of Boussinesq density currents at moderate Reynolds number, and showed that dissipation in the bulk and especially at the interface between the two fluids played only a minor role on the rate of spreading of the current, compared to wall friction. They proposed a semi-empirical formula for the front propagation speed.

It is clear from these observations that resistive effects are at least partly responsible for the discrepancy between theoretical predictions and experimental and numerical results. Birman et al. (2005) calculated the dissipation in lock-exchange flows and observed dissipation to be enhanced near the heavy front. They used free-slip 
conditions at the top and bottom boundaries and thus focused on internal friction without wall friction. It is therefore not possible to compare the relative magnitude of the resistive effects in the bulk vs. those at the walls. Etienne et al. (2005) explored a large range of density ratio $10^{-2} \leqslant \rho_{L} / \rho_{H}<1$ and investigated both free-slip and no-slip boundary conditions. They concluded that, at high density ratio and high Reynolds number, the losses due to boundary-layer friction (and interfacial instability) were small in the dense fluid. Nevertheless, for $R e=1.2 \times 10^{5}$ and $\rho_{L} / \rho_{H}=10^{-2}$ they obtained a heavy front velocity $20 \%$ and $30 \%$ lower than shallow-water predictions (1.3) and (1.1), respectively. Therefore, there is a clear need to clarify the role of resistive effects by disentangling and quantifying the contribution of bulk dissipation from that of wall friction for the complete range of density contrast.

The present investigation aims at improving our understanding of non-Boussinesq density currents through numerical simulations. The primary objective of this work is to study systematically the influence of the resistive effects on the dynamics and structure of density currents. We focus on two-dimensional simulations of lockexchange flows for various Reynolds numbers and density contrasts. The numerical technique employed in this work is a finite-volume volume-of-fluid type method, allowing us to cover the complete range of density contrast $10^{-3} \leqslant \rho_{L} / \rho_{H}<1$ and a wide range of Reynolds number $70 \leqslant R e \leqslant 5 \times 10^{4}$.

We describe the computational approach in $\S 2$ and illustrate the accuracy of the method for describing non-Boussinesq density currents. Then, we focus on the influence of resistive effects on high-Re currents in $\S 3$. First, we show the importance of wall friction in the two asymptotic cases of Boussinesq currents $\left(\rho_{L} / \rho_{H} \approx 1\right)$ and dam break flows $\left(\rho_{L} / \rho_{H} \approx 0\right)$. Then we compare the results for the full range of density contrast with available theories, experiments and numerical simulations. A simple model is proposed for the prediction of the front velocity that includes wall friction. Some interesting results at intermediate Reynolds number are presented in $\S 4$ and a summary of the main results is given in $\S 5$.

\section{Numerical formulation}

\subsection{Basic equations}

We assume the two fluids to be Newtonian, immiscible and incompressible, with no surface tension. The evolution of the two-phase flow is then classically described using the one-fluid formulation of the Navier-Stokes equations, namely

$$
\begin{gathered}
\nabla \cdot \boldsymbol{u}=0, \\
\rho \frac{\partial u}{\partial t}+\rho u \cdot \nabla \boldsymbol{u}=-\nabla p+\rho \boldsymbol{g}+\nabla \cdot\left[\mu\left(\nabla \boldsymbol{u}+{ }^{t} \nabla \boldsymbol{u}\right)\right],
\end{gathered}
$$

where $\boldsymbol{u}, p, \rho$ and $\mu$ are the local velocity, pressure, density and dynamic viscosity in the flow, respectively, and $\boldsymbol{g}$ denotes gravity. In the case of immiscible fluids, the local density obeys

$$
\frac{\partial \rho}{\partial t}+u \cdot \nabla \rho=0 .
$$

In the following, we assume the dynamic viscosity $\mu$ to be a constant and the same for both fluids. Choosing a constant kinematic viscosity instead of a constant dynamic viscosity is expected not to affect the results, provided the density ratio of the two fluids is close to unity and/or the Reynolds number is high. However, this is not true for large density contrasts and low Re. We discuss how this assumption affects the results in Appendix B. We choose the channel height $H$ as length scale, $U=\sqrt{g A t H}$ 


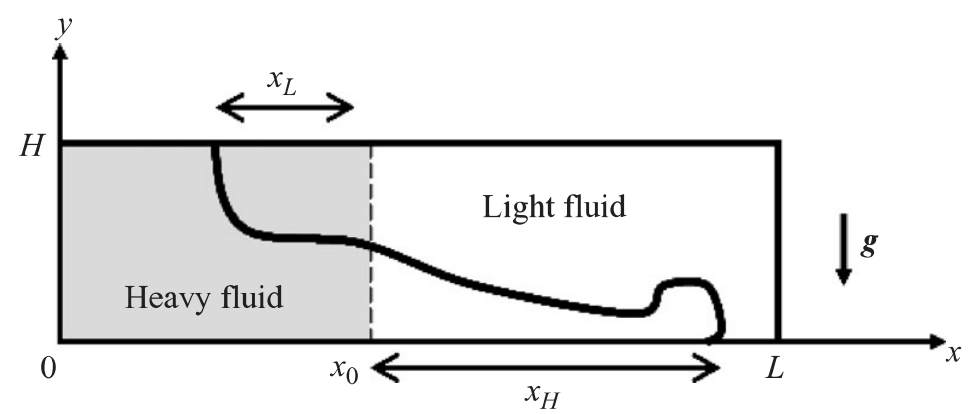

FiguRE 1. The physical configuration and nomenclature used in this work. The dotted line represents the initial separation between the fluids. The thick line represents the separation front at later times.

as velocity scale, $A t=\left(\rho_{H}-\rho_{L}\right) /\left(\rho_{H}+\rho_{L}\right)$ being the Atwood number (Lamb 1945; Gröbelbauer et al. 1993; Seon et al. 2004), and $H / U$ as time scale, with subscripts ' $H$ ' and ' $L$ ' denoting the heavy and light fluid, respectively. The dimensionless density and pressure are given by

$$
\tilde{\rho}=\frac{\rho-\rho_{L}}{\rho_{H}-\rho_{L}}, \quad \tilde{p}=\frac{p}{\rho_{H} U^{2}} .
$$

Note that $\tilde{\rho}$ is the local volume fraction of the heavy fluid. Any variable with a tilde on top is to be understood as dimensionless. The dimensionless form of (2.1)-(2.3) reads

$$
\begin{gathered}
\frac{\partial \tilde{u}_{k}}{\partial \tilde{x}_{k}}=0 \\
\bar{\rho}\left(\frac{\partial \tilde{u}_{i}}{\partial \tilde{t}}+\tilde{u}_{k} \frac{\partial \tilde{u}_{i}}{\partial \tilde{x}_{k}}\right)=\frac{\bar{\rho}}{A t} e_{i}^{g}-\frac{\partial \tilde{p}}{\partial \tilde{x}_{i}}+\frac{1}{R e} \frac{\partial^{2} \tilde{u}_{i}}{\partial \tilde{x}_{k} \partial \tilde{x}_{k}}, \\
\frac{\partial \tilde{\rho}}{\partial \tilde{t}}+\tilde{u}_{k} \frac{\partial \tilde{\rho}}{\partial \tilde{x}_{k}}=0
\end{gathered}
$$

with $\bar{\rho}=(1-A t+2 A t \tilde{\rho}) /(1+A t)$. In addition to the Atwood number, another dimensionless parameter has been introduced in (2.5)-(2.7), namely the Reynolds number defined as

$$
R e=\frac{U H}{v_{H}}=\frac{\sqrt{g A t H} H}{v_{H}},
$$

where $v_{H}$ is the kinematic viscosity of the heavy fluid. Note that the Atwood number is bounded between 0 and 1, with small values corresponding to the Boussinesq limit and large values indicating large density variations. The parameter $\bar{\rho}$ is unity in the Boussinesq limit $(A t \rightarrow 0)$ and reduces to $\tilde{\rho}$ in the non-Boussinesq limit $(A t \rightarrow 1)$. The Reynolds number can be defined based on the heavier or lighter fluid. If the kinematic viscosity is the same in the two fluids, then the two Reynolds numbers match. Instead, if the dynamic viscosity of the two fluids match, the Reynolds number for the heavier fluid will be larger. The difference will be insignificant in the Boussinesq limit, but not when the density difference is large. Here, we choose the larger value and define $R e$ based on the denser fluid, in analogy with the conventional scaling used for the study of gas-liquid flows in which the properties of the heavier phase are used (Bonometti \& Magnaudet 2006).

The physical configuration of the density currents to be considered here is shown in figure 1. Initially, a slab of heavy fluid of density $\rho_{H}$ (shown in figure 1 as the shaded 
region) is separated from the light fluid of density $\rho_{L}$. The heavy fluid is initially of width $\tilde{x}_{0}$ along the flow direction and extends over the entire height $H$ of the channel (lock-exchange configuration). The computational domain is a rectangular box of size $L \times H, L$ and $H$ being the size in the spreading and vertical directions, respectively. The box is typically taken to be very long along the streamwise direction in order to allow free unhindered development of the current. We denote by $\tilde{x}_{L}\left(\operatorname{resp} . \tilde{x}_{H}\right.$ ) the distance crossed by the light (resp. heavy) front as time proceeds.

\subsection{The computational approach}

The numerical technique is a finite-volume/volume-of-fluid method. Equations (2.5)(2.7) are solved using the JADIM code developed at IMFT, Toulouse. This code was originally written for the description of gas-liquid flows and is capable of accurately capturing the dynamics of flows with high density contrasts (Bonometti \& Magnaudet 2006, 2007). Briefly, the momentum equations are discretized on a staggered orthogonal grid using a finite-volume approach. The spatial discretization is performed using second-order centred differences. Time-advancement is achieved through a third-order Runge-Kutta algorithm for advective and source terms and a Crank-Nicolson algorithm for viscous stresses. Incompressibility is satisfied at the end of each time step through a projection method. The algorithm is second-order accurate in both time and space.

In this approach, the equation of evolution of the density is hyperbolic. This is equivalent to choosing a Schmidt number $S c \rightarrow \infty$. Note that although no physical diffusivity is introduced in the advection of $\tilde{\rho}$ (i.e. in (2.7)), the numerical thickness of the interface is not strictly zero as it is typically resolved over three grid cells (Bonometti \& Magnaudet 2007). Therefore, a finite effective Schmidt number can be estimated, which depends somewhat on $R e$ and on the degree of spatial resolution. Based on extensive tests of measurement of the interface thickness, Bonometti \& Balachandar (2008) estimated the effective Schmidt number to be $O\left(10^{3}\right)$.

The transport equation for the density is solved using a modified version of the transport scheme proposed by Zalesak (1979). This scheme belongs to the family of Flux Corrected Transport schemes (Boris \& Book 1973) initially derived for the treatment of shocks. Details of the algorithms used in this code, as well as validations in the case of flows with high density contrast, may be found in Bonometti \& Magnaudet (2007).

We use free-slip conditions at the boundaries along the vertical sidewalls. No-slip conditions (unless otherwise specified) and zero normal gradient for $\tilde{\rho}$ are imposed at the top and bottom walls. The initial density $\tilde{\rho}$ is simply defined as 1 for $\tilde{x} \leqslant \tilde{x}_{0}$ and 0 for $\tilde{x}>\tilde{x}_{0}$ and the fluid is initially at rest. In all the simulations presented here, we use a $1600 \times 160$ grid in a $25 H \times H$ domain (i.e. $L=25 H$ and $H / \Delta z=160$ ), except for the case $\operatorname{Re}=5 \times 10^{4}$ for which a $2400 \times 240$ grid is used $(H / \Delta z=240)$. Several preliminary tests with more refined grids were performed $(H / \Delta z=320)$, and the results to be presented were found to be identical (see figures 2 and 3 for an illustration).

\subsection{Preliminary tests}

The present technique was already used and validated in various problems. It was shown to reproduce correctly the dynamics of bubbly flows at low-to-moderate $R e$ (Bonometti \& Magnaudet 2006, 2007) as well as planar density currents for a wide range of $R e$ in the Boussinesq limit (Bonometti \& Balachandar 2008). In order to check the capability of the code to simulate density currents with high density ratios, two additional sets of validation were considered: the first one aims at reproducing a 


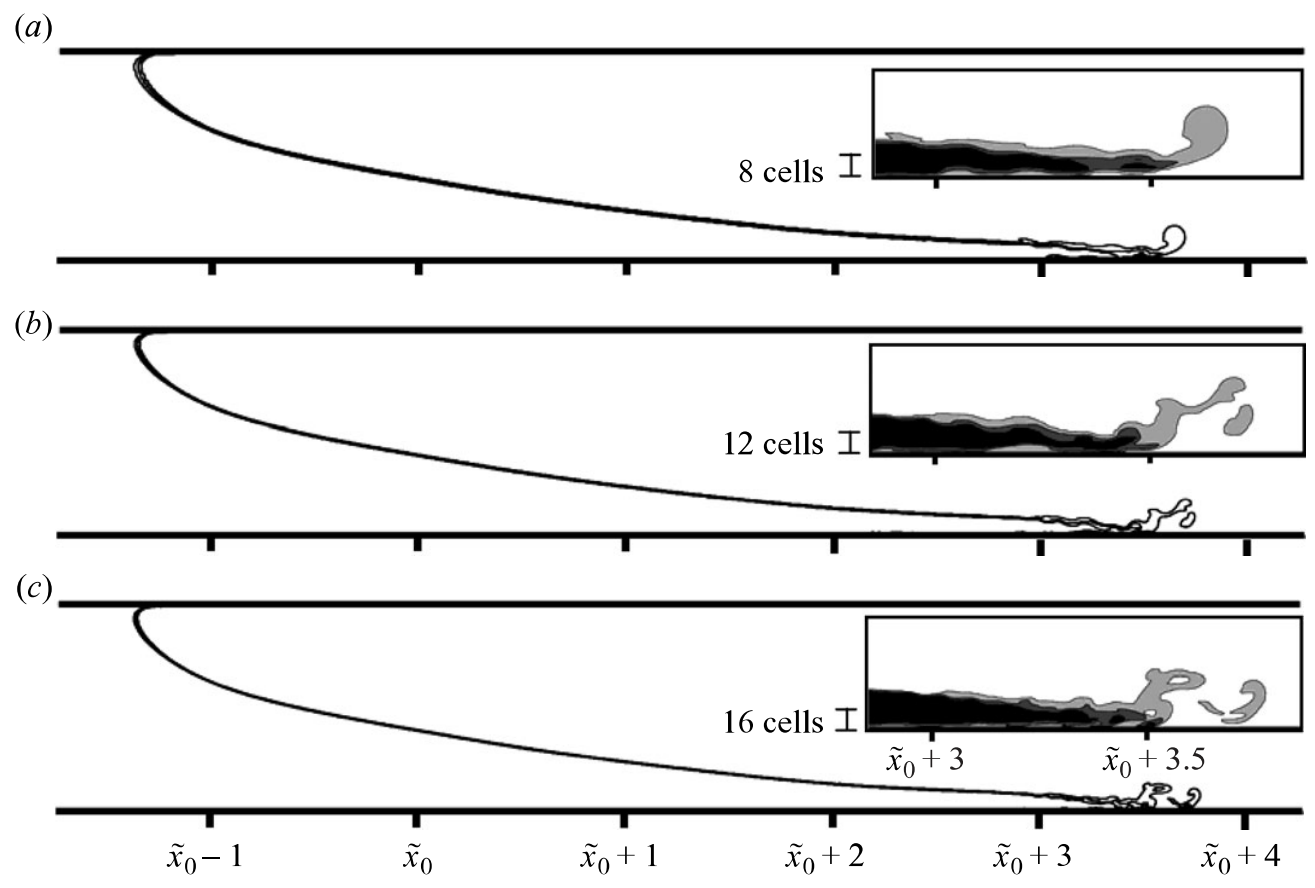

FIGURE 2. Iso-contours of $\tilde{\rho}$ at $\tilde{t}=2.8\left(\rho_{L} / \rho_{H}=10^{-3}, R e=5 \times 10^{4}\right)$. (a) Coarse grid $H / \Delta z=$ 160, (b) medium grid $H / \Delta z=240$, (c) fine grid $H / \Delta z=320$. Values of plotted iso-contours are $\tilde{\rho}=0.05-0.5-0.95$. Note that except in the vicinity of the heavy current head, they are almost undistinguishable because of the high Schmidt number. Inset: close-up of the heavy current head. The size of the close-up windows is $H \times 0.25 H$. Different levels of grey are used to show iso-contour locations better.

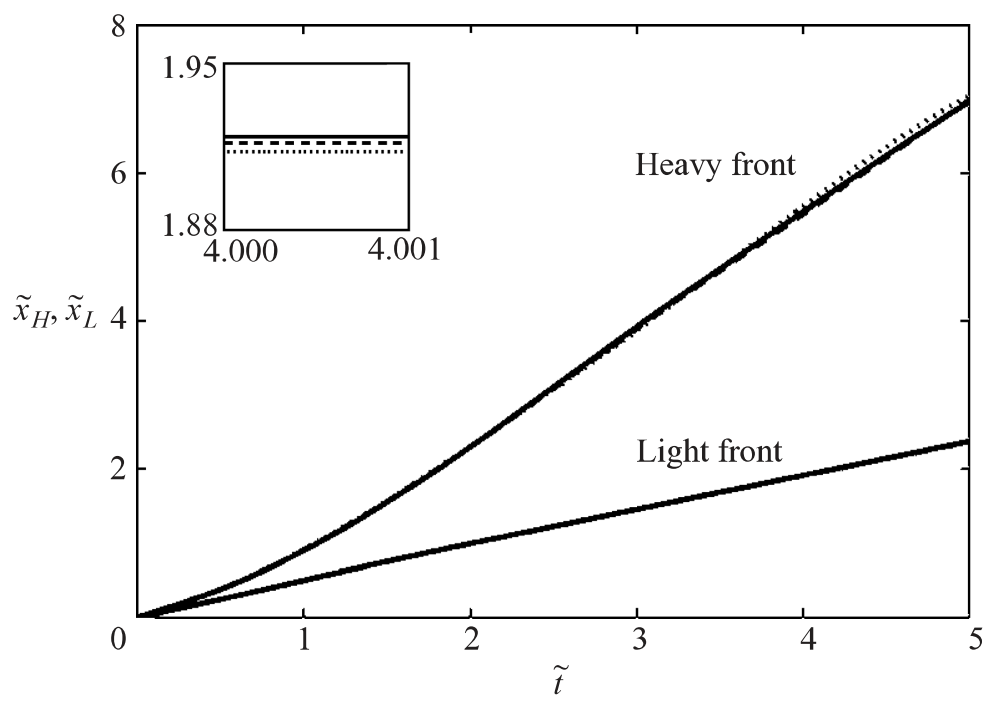

FIGURE 3. Time evolution of the position of the light and heavy fronts $\left(\rho_{L} / \rho_{H}=10^{-3}, R e=\right.$ $5 \times 10^{4}$ ). Dotted line, coarse grid $H / \Delta z=160$; dashed line, medium grid $H / \Delta z=240$; solid line, fine grid $H / \Delta z=320$.

recent computational result obtained using a spectral method by Birman et al. (2005), while the second provides a test of spatial resolution. In the first test, the comparison of density contours, temporal evolution of the front velocity and viscous dissipation inside the current as computed in the two different numerical methods, revealed a very good agreement (results are not shown here).

We then consider the second test with a high density contrast of $\rho_{L} / \rho_{H}=10^{-3}$ and a high Reynolds number of $R e=5 \times 10^{4}$. The longitudinal domain size is $L=13.5 \mathrm{H}$ and 
the location of the initial lock is $\tilde{x}_{0}=3.25$. No-slip boundary conditions are imposed at the top and bottom walls. The computational grid is varied from $832 \times 160$ to $1664 \times 320(H / \Delta z$ is varied from 160 to 320$)$.

Density contours at time $\tilde{t}=2.8$ are plotted in figure 2 and the position of the heavy and light front as a function of time is plotted in figure 3 . The front locations are determined by using the vertically integrated dimensionless height $\tilde{h}$ defined as (Shin et al. 2004; Marino et al. 2005; Birman et al. 2005)

$$
\tilde{h}(\tilde{x}, \tilde{t})=\int_{0}^{1} \tilde{\rho} \mathrm{d} \tilde{z} .
$$

The quantity $\tilde{x}_{H}(t)$ is then defined as the location where $\tilde{h}$ becomes smaller than a small threshold $\delta$ and $\tilde{x}_{L}(t)$ as the location where $\tilde{h}$ becomes greater than $1-\delta$. Here we use $\delta=0.01$ (see Cantero et al. 2006 for a discussion of the choice of this parameter).

The shape of the current is typical of highly contrasted density currents, i.e. the height of the heavy front is small and slender while the height of the light front is half of the channel approximately. In contrast to Boussinesq currents, the flow is clearly not symmetric. Although no physical diffusivity is introduced in the advection of $\tilde{\rho}$ in the finite-volume code, the interface is numerically resolved over three grid cells. Thus, small-scale structures continue to become finer with increased resolution. This behaviour is evident in the close-ups in figure 3 (the iso-densities shown are $0.05,0.5$ and 0.95 , respectively). As shown in the figure, the level of resolution of the heavy front increases from 8 to 16 grid points. The global position of the head is mostly insensitive to the spatial resolution. The flow at the heavy front at this Reynolds number is chaotic and thus instantaneous views at different resolution are not expected to be precisely identical. The features of the heavy front obtained at the two highest resolutions are quite comparable. We also looked at the thickness of the light fluid film underneath the propagating heavy fluid. Measurements of the position of some specific density contours in the proximity of the bottom boundary, just behind the heavy current's head, were performed. By averaging the vertical position of the density-contour $\tilde{\rho}=0.5$ behind the head of the current to estimate the thickness of the trapped light fluid film (averaged over a distance equivalent to one initial height $H$ ), we observe that the thickness of the trapped light fluid film is very similar for all spatial resolutions with differences of less than a few per cent.

As shown in figure 3, the evolution of the heavy and light fronts is captured with all resolutions. The front positions computed on the two most refined grids are almost undistinguishable. We also computed the temporal evolution of the total kinetic energy, potential energy and dissipation in the flow for the three resolutions. The variation of the overall energy remains negligibly small during all the simulation. For instance, the variation of the total energy at time $\tilde{t}=5$ is less than $1.6 \%, 1.2 \%$ and $0.8 \%$ for grids $832 \times 160,1248 \times 240$ and $1664 \times 320$, respectively. This test establishes the adequacy of the grid resolution and the accuracy of the code in all cases to be discussed here.

\section{High-Re currents}

\subsection{Shape of density currents}

Figure 4 shows iso-contours of the density field of currents with density contrasts ranging from $\rho_{L} / \rho_{H}=10^{-3}$ to 0.99 . At low density contrast, strong vortex structures are observed in the interface region, while the heads of the light and heavy fronts are 


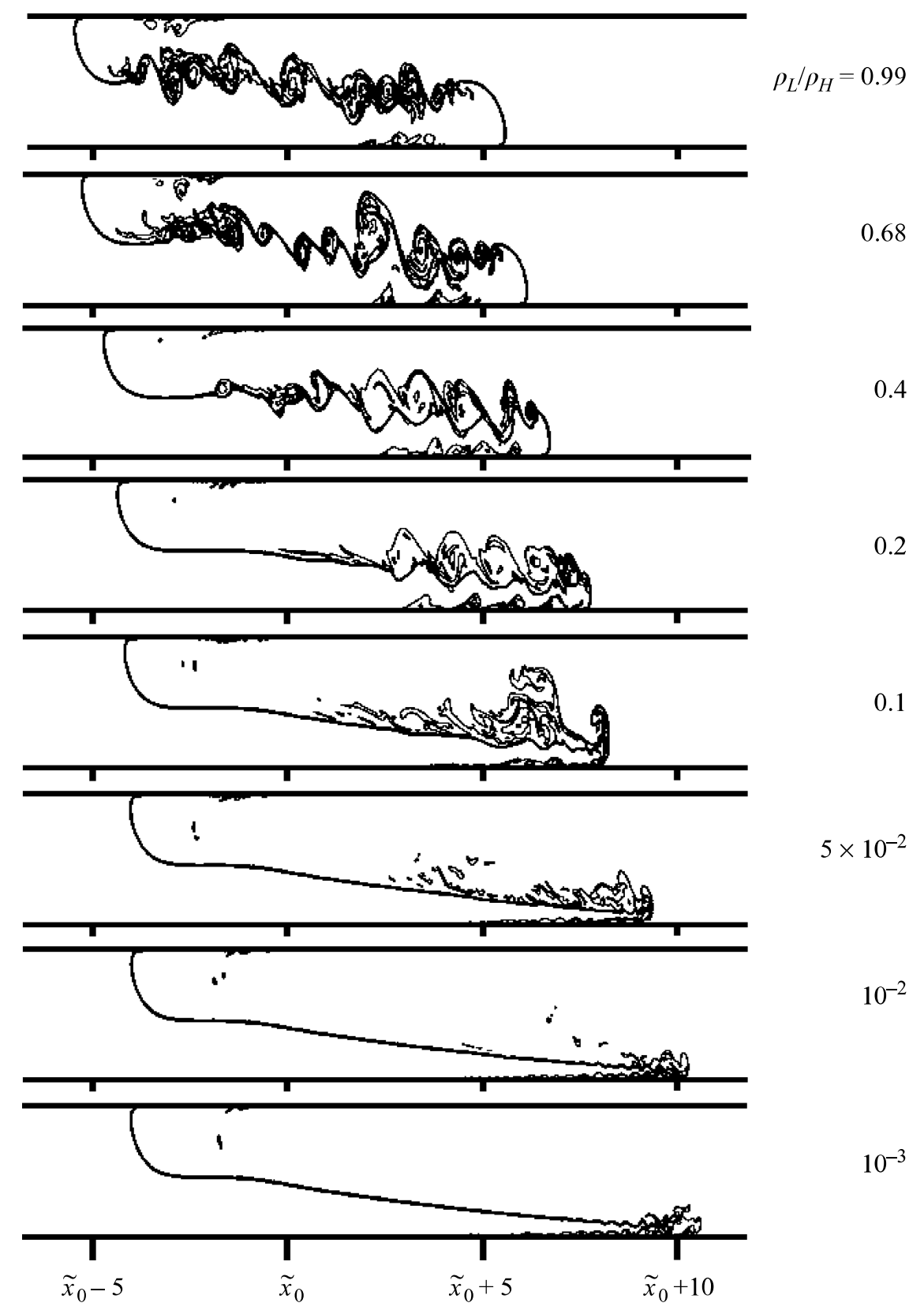

FIGURE 4. Iso-contours of $\tilde{\rho}$ at $\tilde{t}=9(R e=7071)$. Values of plotted iso-contours are $\tilde{\rho}=0.05-0.275-0.5-0.725-0.95$.

smooth. With increasing density contrast, the size of these vortex structures decreases and their location shifts toward the heavy front, in agreement with Benjamin's (1968) stability analysis and with the observations of Etienne et al. (2005). For instance, vortex structures occupy approximately $75 \%, 60 \%, 40 \%, 10 \%$ of the total length of the interface at density contrasts of $\rho_{H} / \rho_{L}=0.99,0.4,0.1,10^{-2}$, respectively. In addition, the characteristic size of the vortex structures decreases by one order of magnitude from the Boussinesq limit to the highly contrasted case. For $\rho_{L} / \rho_{H} \leqslant 10^{-2}$, the tiny vortical structures are confined to the very front of the heavy current, while the light front and the bulk of the interface remain smooth.

The evolution of the vortex structures with the density contrast results from the competition between the free shear generated at the interface of the two fluids and the stratification effects. While shear is of the same order of magnitude for all cases, the stratification effect increases by several orders of magnitude as $\rho_{L} / \rho_{H}$ decreases 
from 0.99 to $10^{-3}$. The Richardson number, defined as $R i=-g(\partial \rho / \partial z) / \rho(\partial u / \partial z)^{2}$, compares these two effects. As shown in Bonometti \& Balachandar (2008), it can be estimated as

$$
R i \sim \sqrt{\frac{S c}{R e}}\left(1+\frac{\rho_{H}}{\rho_{L}}\right) .
$$

It is clear from this scaling that the stratification is much larger for high density ratio. Asymptotically, the Boussinesq and the highly contrasted cases might be qualified as 'pure shear regime' and 'pure stratification regime', respectively. Note that the shape of the highly contrasted current in figure 4 is in qualitative agreement with the experimental observations of Martin \& Moyce (1952) and figure 13(c) of Lowe et al. (2005).

\subsection{Front velocity}

The importance of wall friction on the evolution of Boussinesq density currents has been considered over the last decade (Härtel et al. 2000; Necker et al. 2005). Härtel et al. (2000) computed two-dimensional Boussinesq lock-exchange flows for $O\left(10^{2}\right) \leqslant R e \leqslant O\left(10^{5}\right)$ with both free-slip and no-slip boundary conditions. They observed that the front velocity of density currents spreading over a no-slip boundary is reduced by about $5 \%$ at $R e=O\left(10^{5}\right)$ and about $25 \%$ at $R e=O\left(10^{2}\right)$. They concluded that no qualitative difference in the flow structure could be observed between the two boundary conditions. However, for fluids of moderate density contrast, Birman et al.'s (2005) results showed differences of up to $30 \%$ between front velocities for both the light and heavy fluids at $R e=3381$. Therefore, there is a need to clarify the role played by wall friction on the shape of density currents and also to quantify its impact on the front velocity in the Boussinesq limit as well as in contrasted flows. In this section, we provide evidence that the friction at walls can significantly modify the structure and the dynamics of contrasted density currents.

\subsubsection{The Boussinesq lock-exchange flow}

Let us first revisit the Boussinesq lock-exchange problem and consider the effect of friction at the walls on the front velocity. In the Boussinesq limit, the velocities of the heavy and light fronts are similar and the temporal evolution of the front location of a high-Re Boussinesq density current is depicted in figure 5. Along with the present results, we also include experimental results of lock-exchange flows for pairs of gases and pairs of liquids, respectively, and the results from a spectral simulation (see table 1). Note that in experiments, because of difficulties in approximating the instantaneous and clean lifting of the lock, it is hard to pin down the exact starting time (Martin \& Moyce 1952). So the experimental data have been shifted to impose a fixed current location at $\tilde{t}=4$ as reference. It must be stressed that this adjustment does not alter the overall results, since we focus on the variation of the front position (i.e. the spreading rate) rather than the exact position of the front at a specific time. The slope of the dotted line is that of Benjamin's solution (1.2).

The evolution of the front on the free-slip wall closely follows Benjamin's halfdepth dissipationless speed result to within $0.5 \%$. Note that Härtel et al. (2000) also computed Boussinesq currents spreading on free-slip walls at $R e \geqslant 6300$ and found the difference between the computed front velocity and Benjamin's prediction to be less than $2 \%$. The close agreement between the theory and the computed free-slip results supports the view that in the Boussinesq limit, effects of large-scale vortical structures and mixing at the interface on the speed of propagation of the currents is minimal. 
Reference

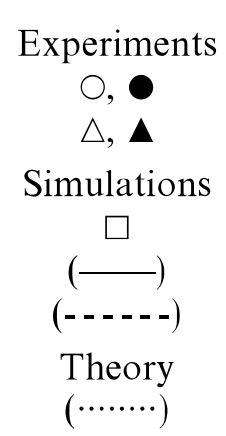

Shin et al. (2004)

Gröbelbauer et al. (1993)

Cantero et al. (2007)

Present results with no-slip boundary condition

Present results with free-slip boundary condition

Benjamin (1968)
$\rho_{L} / \rho_{H}$

Re

$\begin{array}{rr}0.993 & 1.66 \times 10^{4} \\ 0.901 & 9.3 \times 10^{3}\end{array}$

$\approx 1$

$6.33 \times 10^{3}$

0.99

$5 \times 10^{4}$

0.99

$5 \times 10^{4}$

$\approx 1$

$\infty$

TABLE 1. Data used in figure 5 to study the spreading rate of Boussinesq currents.

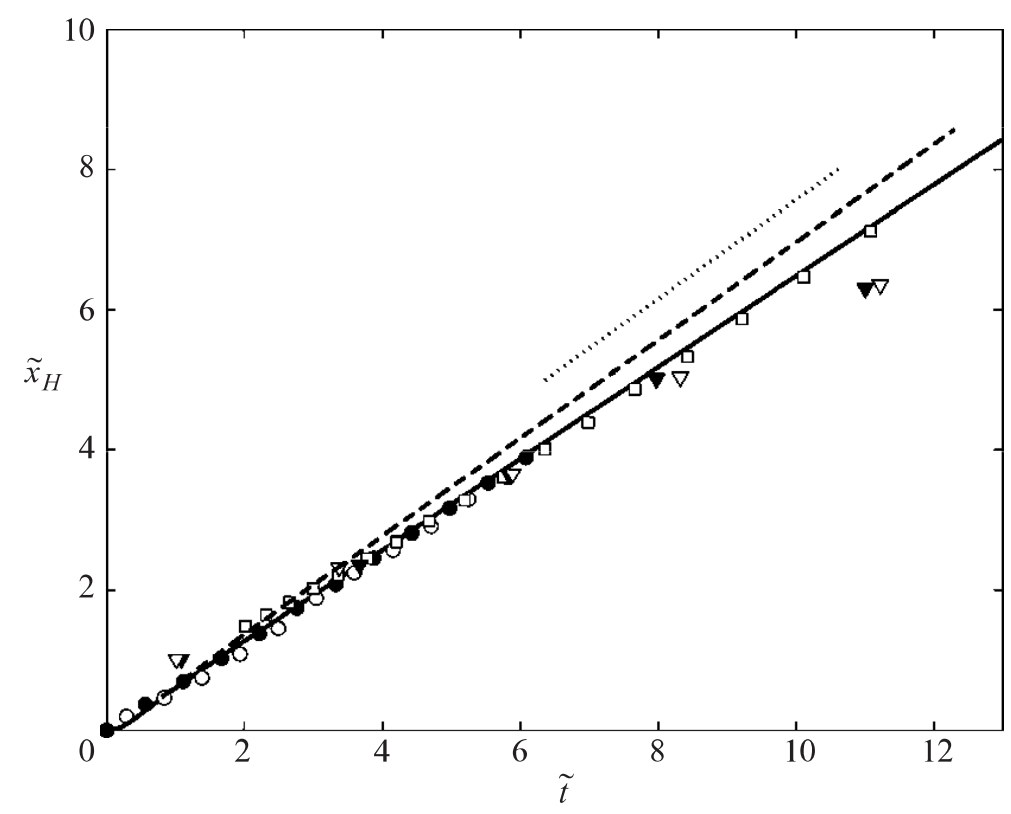

Figure 5. Time evolution of the front position of high-Re Boussinesq density currents. The experimental results have been shifted in time so that $\tilde{t}=4$ is the reference time. See table 1 for key.

When wall friction is taken into account, all the experimental and numerical data slightly, but noticeably, depart from the theory. The evolution of the computed current spreading on a no-slip wall agrees well with available experimental and numerical data. The discrepancy between theoretical (free-slip) and no-slip front velocities is observed to be in the range 5-15\%.

\subsubsection{The dam-break flow}

We now consider the temporal evolution of the front position of highly contrasted density currents. For the heavy fluid, this configuration corresponds to the dam-break problem whereas for the light fluid spreading under a rigid boundary this corresponds to a cavity flow. The temporal evolution of the heavy front is presented in figure 6, which also includes available experimental and numerical data, as well as theoretical predictions (see table 2). Again the data have been shifted to overlap the position of the heavy front at $\tilde{t}=4$ as reference (Martin \& Moyce 1952).

Immediately after release, the heavy current can be seen to go through an acceleration phase where the velocity of the front rapidly increases. Subsequently, 


\begin{tabular}{|c|c|c|c|c|c|}
\hline & Reference & $\operatorname{Re}$ & & Reference & Re \\
\hline$\underset{\Delta}{\text { Experiments }}$ & $\begin{array}{l}\text { Martin \& Moyce } \\
\quad(1952)\end{array}$ & $4 \times 10^{4}$ & $\begin{array}{l}\text { Simulations } \\
(\longleftarrow)\end{array}$ & $\begin{array}{l}\text { Present results with no- } \\
\text { slip boundary condition }\end{array}$ & $5 \times 10^{4}$ \\
\hline$\nabla, \mathbf{\square}$ & $\begin{array}{l}\text { Martin \& Moyce } \\
(1952)\end{array}$ & $1.2 \times 10^{5}$ & $(-----)$ & $\begin{array}{l}\text { Present results with free- } \\
\text { slip boundary condition }\end{array}$ & $5 \times 10^{4}$ \\
\hline$\square$ & Dressler (1954) & $3.2 \times 10^{5}$ & Theories & & \\
\hline$\bullet$ & $\begin{array}{l}\text { Lauber \& Hager } \\
\text { (1998) }\end{array}$ & $O\left(10^{6}\right)$ & $(-\cdot \cdot \cdot \cdot \cdot \cdot \cdot)$ & $\begin{array}{l}\text { One-layer shallow- } \\
\text { water, (1.1) }\end{array}$ & $\infty$ \\
\hline 0 & $\begin{array}{l}\text { Stansby et al. } \\
\text { (1998) }\end{array}$ & $7 \times 10^{5}$ & $(\cdots \cdots \cdots)$ & $\begin{array}{l}\text { Two-layer shallow-water, } \\
\text { Lowe et al. (2005) }\end{array}$ & $\infty$ \\
\hline
\end{tabular}

TABLE 2. Data used in figure 6 to study the spreading rate of non-Boussinesq currents. Note that for the present results $\rho_{L} / \rho_{H}=10^{-3}$.

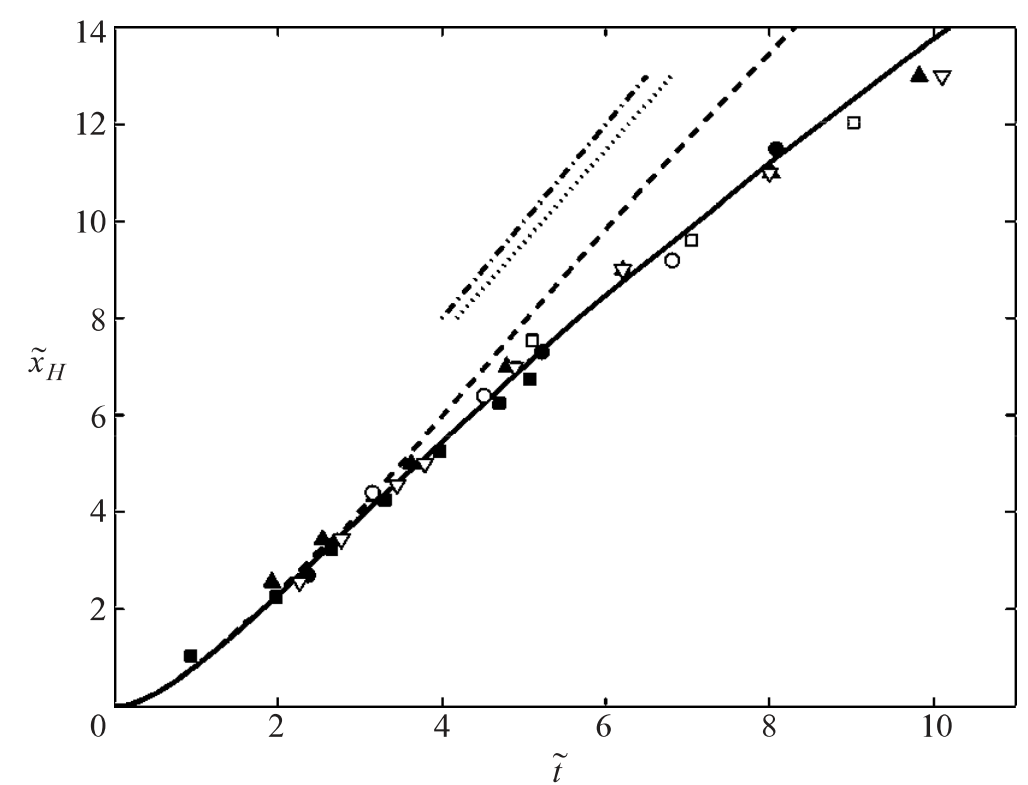

FigurE 6. Time evolution of the front position of high-Re non-Boussinesq density currents with $\rho_{L} / \rho_{H}=O\left(10^{-3}\right)$. The experimental results have been shifted in time so that $\tilde{t}=4$ is the reference time. See table 2 for key.

the heavy front reaches a near constant velocity, which for the free-slip condition is in good agreement with shallow-water predictions. Note that the difference in the predicted velocity of the heavy front from the one-layer and two-layer shallow-water theories is small. Later, in figure 9, it will be shown that the velocity of the light front is also in close agreement (within $0.5 \%$ ) with the theoretical prediction $(1.2 b)$.

As already observed for Boussinesq density currents, the experimental and numerical data which include wall friction effects depart from results based on inviscid theories. The evolution of the computed heavy current spreading on a no-slip wall agrees well with available experimental data. Note that this conclusion is in qualitative agreement with the results of Birman et al. (2005) who observed the speed of the front on a no-slip wall to be slower by about $30 \%$ than that on a free-slip wall in a current of modest density contrast $\left(\rho_{L} / \rho_{H}=0.4\right.$ at $\left.R e=3381\right)$.

The effect of wall friction at a larger density contrast has been considered by Etienne et al. (2005). A comparison of the time evolution of the heavy and light fronts spreading on free-slip and no-slip walls for a density contrast of $\rho_{L} / \rho_{H}=4.6 \times 10^{-2}$ 
(a)

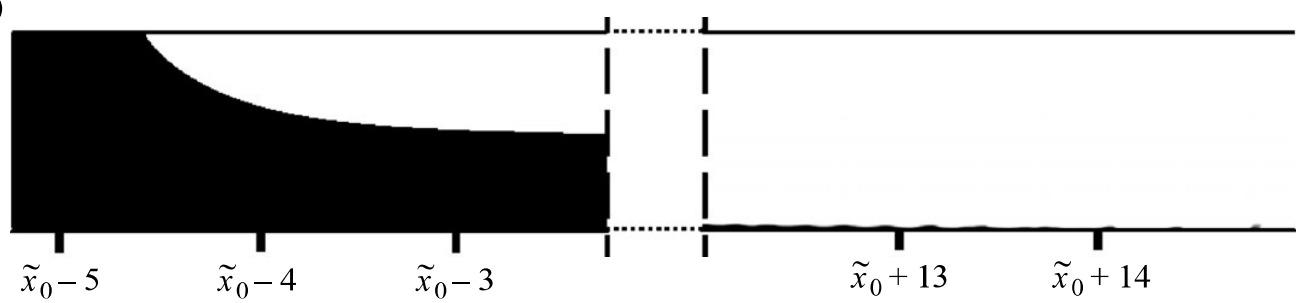

(b)

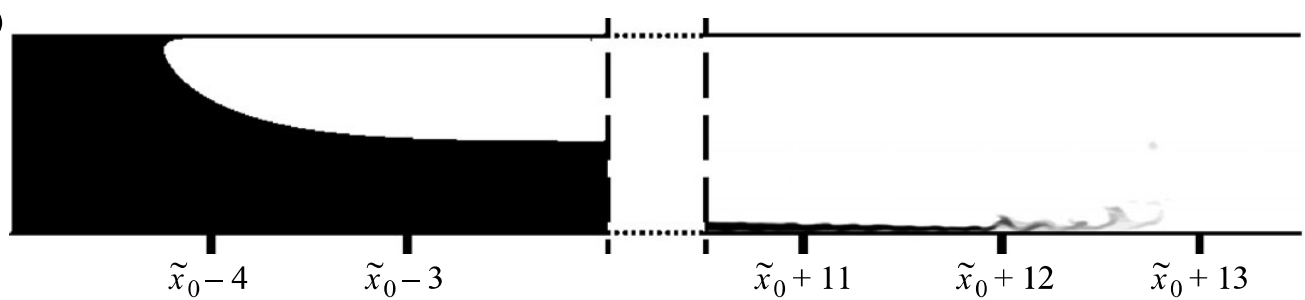

FiguRE 7. Shadowgraphs of the light and heavy high-Re fronts at $\tilde{t}=9\left(\rho_{L} / \rho_{H}=10^{-3}, R e=\right.$ $\left.5 \times 10^{4}\right)$. Here, we imposed $(a)$ a free-slip and $(b)$ a no-slip boundary condition at the top and bottom walls.

(their figure 9) showed little difference in the current velocities, as the currents accelerated to a downstream distance of $2.5 \mathrm{H}$, which is approximately equivalent to a dimensionless time $\tilde{t}=2.5$. This is in agreement with the results presented in figure 6 , where we found the velocity of the heavy front with free-slip and no-slip boundaries to be nearly the same during the acceleration phase $(\tilde{t}<3)$. During this acceleration period, the boundary layer resulting from the no-slip condition is under development. Hence, the effect of wall friction remains weak and the evolution of the front velocity is expected to be little affected by the wall boundary condition, as observed by Etienne et al. (2005). However, at later times, the velocity of the front is significantly slowed down by wall friction.

For a modest density contrast, Birman et al. (2005) reported that the structure of the heavy and light fronts remained qualitatively the same and did not significantly vary with the wall boundary condition. The shape of the computed light and heavy fronts at a large density contrast of $\rho_{L} / \rho_{H}=10^{-3}$ and $R e=5 \times 10^{4}$ is shown in figure 7 for both wall conditions. This figure indicates that the qualitative observations of Birman et al. (2005) are appropriate even at large density contrasts. Although subtle, a thin interstitial film of heavy fluid along the top wall above the propagating light current and a thin undulating layer of light fluid along the bottom wall below the advancing heavy current can be observed in the case of no-slip boundaries. These films modify the shape of the light and heavy fronts. For instance, the shape of the light front in the case of a no-slip wall is more rounded and not as sharp in its approach to the wall as in the free-slip case. As for the heavy current, the no-slip bottom boundary results in the unstable situation of a layer of heavy fluid riding on top of a thin layer of light fluid. The resulting instability can be observed in figure 7 through the undulating nature of the heavy front. A similar Rayleigh-Taylor instability occurs at the light front as well; its effect is more clearly seen in figure 4 as the unstable drops of heavy fluid falling from the top wall. In addition to these instabilities, the presence of the thin films along the top and bottom walls leads to a slight displacement of the near-horizontal interface downstream and upstream of the head of the light and heavy fronts, respectively, thus increasing the overall height of the currents by a few per cent. 


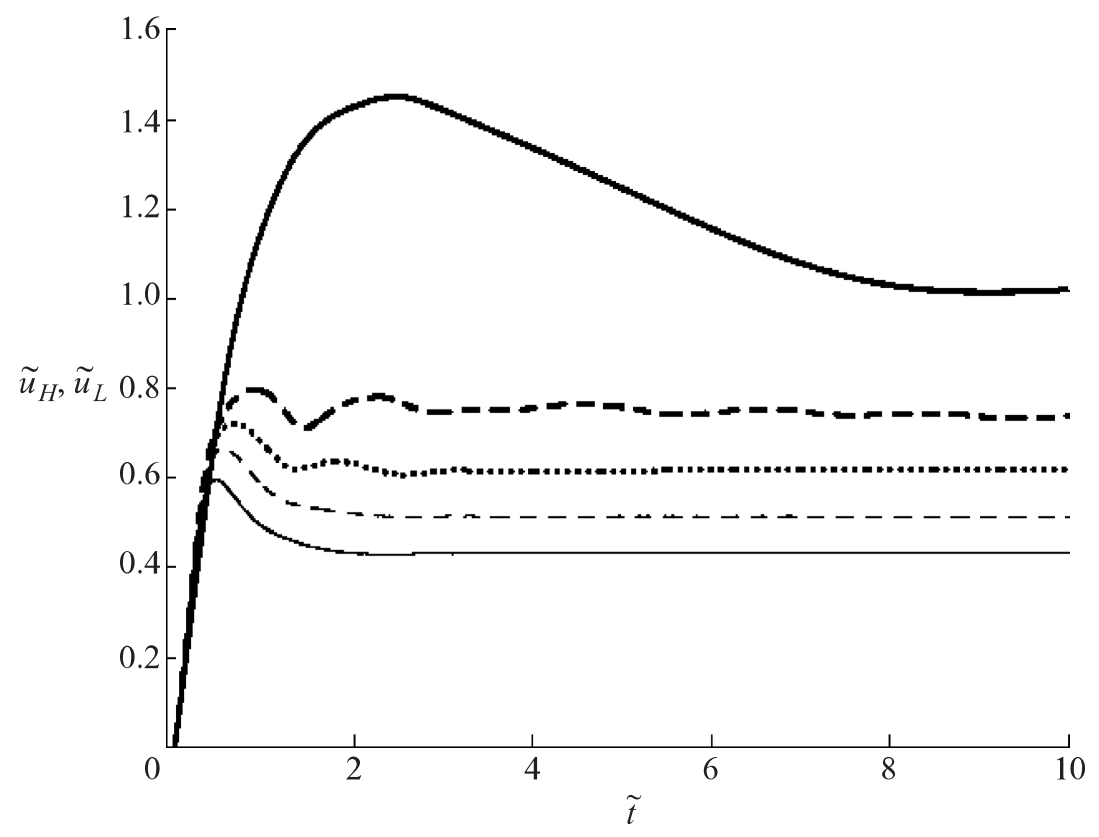

FIGURE 8 . Time evolution of the heavy front velocity $\tilde{u}_{H}$ (thick line) and light front velocity $\tilde{u}_{L}$ (thin line). The dotted, dashed and solid lines correspond to $\rho_{L} / \rho_{H}=0.99,0.4$ and $10^{-3}$, respectively. The Reynolds number of the flow is $R e=7071$.

\subsubsection{Currents of arbitrary density contrast}

The temporal evolutions of the heavy and light front velocities are depicted in figure 8 for the two limit cases $\rho_{L} / \rho_{H}=10^{-3}$ and $\rho_{L} / \rho_{H}=0.99$ and for the intermediate case $\rho_{L} / \rho_{H}=0.4$ (note that in order to keep the Reynolds number constant while the density ratio varied, we modified the viscosity of the fluids accordingly). Velocities of the light front $\tilde{u}_{L}$ and of the heavy front $\tilde{u}_{H}$ are computed as $\tilde{u}_{L}=\left|\mathrm{d} \tilde{x}_{L} / \mathrm{d} \tilde{t}\right|$ and $\tilde{u}_{H}=\mathrm{d} \tilde{x}_{H} / \mathrm{d} \tilde{t}$. Increasing the density contrast of the current is observed to increase the gap between the light and heavy front velocities. For instance, for $\tilde{t}=10$, we find $\tilde{u}_{H} / \tilde{u}_{L} \approx 1,1.44$ and 2.35 for $\rho_{L} / \rho_{H}=0.99,0.4$ and $10^{-3}$, respectively. The transient time of the heavy current, measured as the dimensionless time at which the current reaches its maximum velocity, strongly increases with increasing density contrast, whereas the transient time of the light current slightly decreases with increasing density contrast. In dimensionless units, the transient time of the heavy current is 0.7 and 2.5 at $\rho_{L} / \rho_{H}=0.99$ and $10^{-3}$, respectively, whereas the corresponding transient time of the light current is 0.7 and 0.5 , respectively. A possible explanation could be found in the time required to reach the final shape of the current heads. With increasing density contrast, the height of the light current remains constant within a few per cent. However, a close look reveals that at higher density contrasts, the final shape of the light fluid head is closer to its initial shape, so that the transient time decreases. In contrast, the head of the heavy current becomes increasingly slender, departs from the initial condition and requires more time to reach the steady state with increasing density contrast.

The velocities of the light and heavy fronts of the density currents are compared with available analytical, experimental and numerical data in figure 9 for a wide range of density contrasts (see table 3). The theories include Benjamin's half-depth dissipationless speed result (equation (1.2)), the one-layer shallow-water theory ((1.3), Ungarish 2007) and the two-layer shallow-water theory (see e.g. equations 3.11-12 in Lowe et al. 2005). Note that these theories predict the same velocity for the light 


\section{Reference}

Experiments

$\begin{array}{cc}\bigcirc & \text { Keller \& Chyou }(1991) \\ & O\left(10^{3}\right) \leqslant R e \leqslant O\left(10^{4}\right) \\ \square & \text { Gröbelbauer } \text { et al. }(1993) \\ & O\left(10^{4}\right) \leqslant \operatorname{Re} \leqslant O\left(10^{5}\right) \\ \diamond & \text { Lowe } \text { et al. }(2005) \\ & 1.5 \times 10^{4} \leqslant \operatorname{Re} \leqslant 1.5 \times 10^{5}\end{array}$

Simulations

$\begin{array}{ll}\text { is } & \text { Birman } \text { et al. }(2005) \\ & 3 \times 10^{3} \leqslant R e \leqslant 1.1 \times 10^{4} \\ \nabla & \text { Present results with free-slip } \\ & \text { boundary condition } R e=7071 \\ \triangle & \text { Present results with no-slip } \\ & \text { boundary condition } R e=7071\end{array}$

\section{Reference}

Theories

$(\square$ Benjamin's result (1968)

(…....) Ungarish (2007) one-layer shallow-water

(-..--) Lowe et al. (2005) two-layers shallow-water

(-......) Present model (3.2) (with $k=0.6$ )

$\times \quad$ Etienne et al. (2005) $1 \times 10^{4} \leqslant \operatorname{Re} \leqslant 3.4 \times 10^{6}$

Present results with free-slip boundary condition $R e=5 \times 10^{4}$ Present results with no-slip boundary condition $R e=5 \times 10^{4}$

TABLE 3. Data used in figure 9. Note that the $\nabla$ and $\boldsymbol{\nabla}$ symbols are superimposed in the figure.
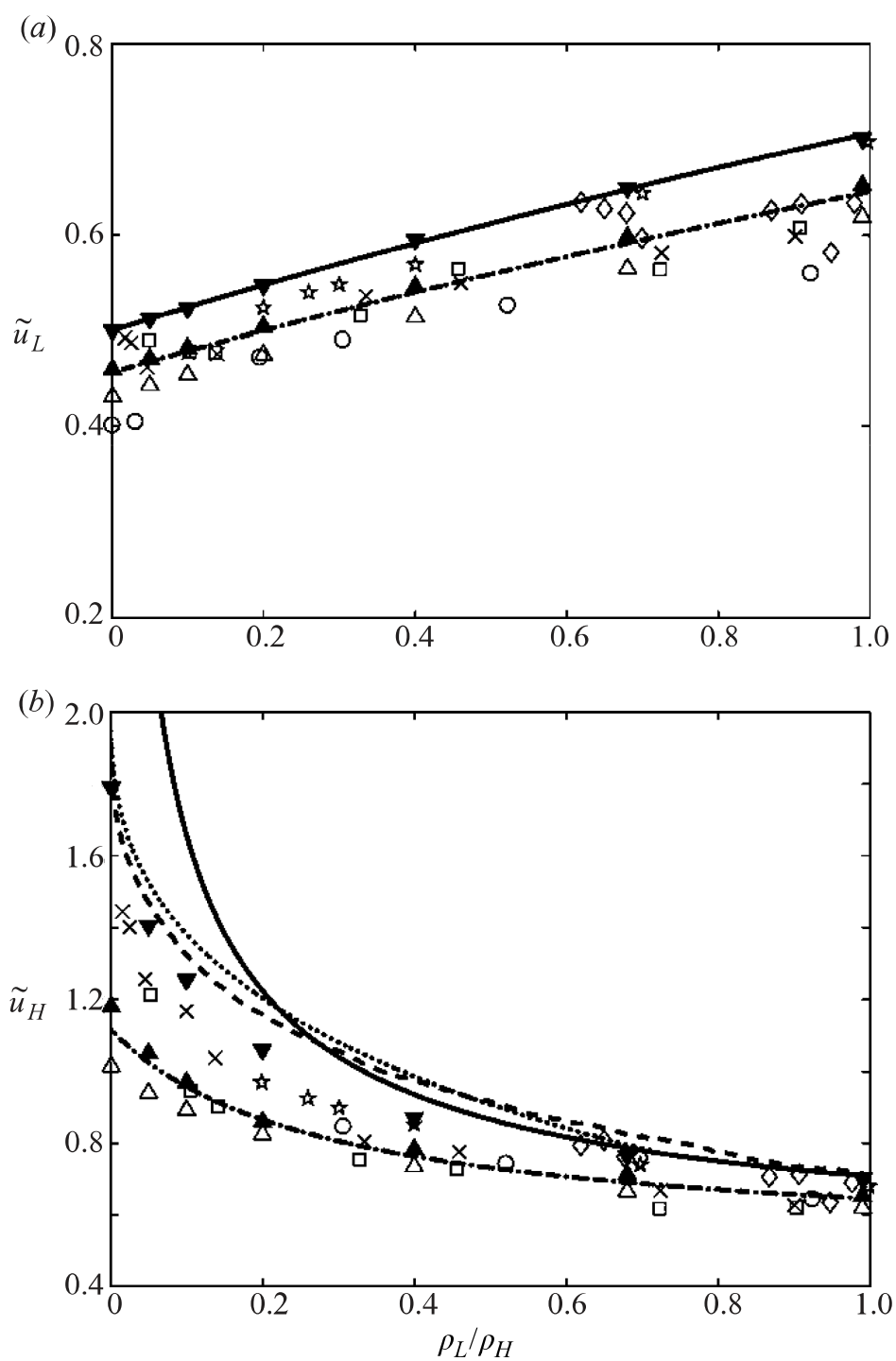

FIGURE 9. Variation of the speed of the light and heavy fronts with the density contrast. See table 3 for key. 
front. As for the heavy front velocity, the theories agree within about $5 \%$ for currents of density contrast in the range $0.25 \leqslant \rho_{L} / \rho_{H} \leqslant 1$ (figure $9 b$ ). For more contrasted currents, Benjamin's prediction becomes singular and gives much higher values than the shallow-water theories whose results are relatively similar. This is due to the assumption of energy conservation implicit in Benjamin's speed result (1.2), which becomes increasingly inappropriate for small $\rho_{L} / \rho_{H}$ (Ungarish 2007).

The present computed velocities agree reasonably well with other experimental and numerical results. Most of the experimental data appear to be bounded by the velocities obtained in the case of free-slip (upper limit) and no-slip (lower limit) boundaries. Over the entire range of density contrast, the computed velocities of the light front obtained with free-slip boundary conditions at $R e=5 \times 10^{4}$ agree to within $0.5 \%$ with Benjamin's prediction, whereas the results obtained with no-slip boundary conditions are approximately $8 \%$ below the theoretical prediction.

The velocity of the heavy front obtained with a free-slip boundary condition is found to be in good agreement with Benjamin's half-depth dissipationless speed result only for small density contrasts $\left(0.6 \leqslant \rho_{L} / \rho_{H} \leqslant 1\right)$. With further increase in density contrast $\left(0.1 \leqslant \rho_{L} / \rho_{H} \leqslant 0.6\right)$, the results start to depart from Benjamin's prediction as well as from one- and two-layer shallow-water theories, the maximum discrepancy being less than $10 \%$.

Additional simulations were performed for the two specific intermediate density ratios of $\rho_{L} / \rho_{H}=0.2$ and 0.4 , for which the difference between the speed of the heavy front and that predicted by the shallow-water theory is more pronounced. First, we performed computations in the range $7071 \leqslant R e \leqslant 5 \times 10^{4}$, with free-slip conditions at the top and bottom boundaries. Results are shown in figure 9 (as the solid and open downward pointing triangles). It is observed that the front velocities are identical to within a few per cent. Additional simulations at varying resolution (similar to those discussed in figure 2) show that the velocity of the heavy front at all the Reynolds numbers presented here is numerically well converged. These extra computations allow us to conclude that the difference between the front velocity predicted by shallow-water theory and the present computations with free-slip boundaries is due neither to the finite value of the Reynolds number, nor to spatial resolution.

In this case, we further investigated the effect of three-dimensionality of the flow on the front velocity. We thus performed a three-dimensional simulation of the configuration $\left(\rho_{L} / \rho_{H}=0.2 ; R e=7071\right)$ in addition to the present two-dimensional simulations. Because of computational resource limitations, the spatial resolution used for the three-dimensional simulation is lower than that used for the two-dimensional simulations. We use a grid of $480 \times 60 \times 80$ points along the streamwise, spanwise and wall-normal directions $(x, y, z)$ with a domain of size $15 H \times 1.5 H \times H$. This resolution is half that used in figure 2(a) (i.e. $H / \Delta z=80$ ). The temporal evolution of the front position was observed to be almost identical to within a few percent between the two- and three-dimensional simulations.

We conjecture that the discrepancy between the simulations with free-slip boundary conditions and the shallow-water theory at intermediate density ratio is due to the dissipation at the interface which plays a role, although subtle, on the heavy front velocity at intermediate density ratios, say $0.1 \leqslant \rho_{L} / \rho_{H} \leqslant 0.6$. First, in this range of parameters, the mixing at the interface is observed to take place close to the heavy front (figure 4), and leads to small periodic oscillations in the front velocity (see the dashed curve of figure 8 for an illustration). Secondly, by looking at the dissipation at the interface along the body of the current for three density contrasts $\rho_{L} / \rho_{H}=0.99$, 0.2 and $10^{-3}$, respectively, we observe that the location of the maximum dissipation is 

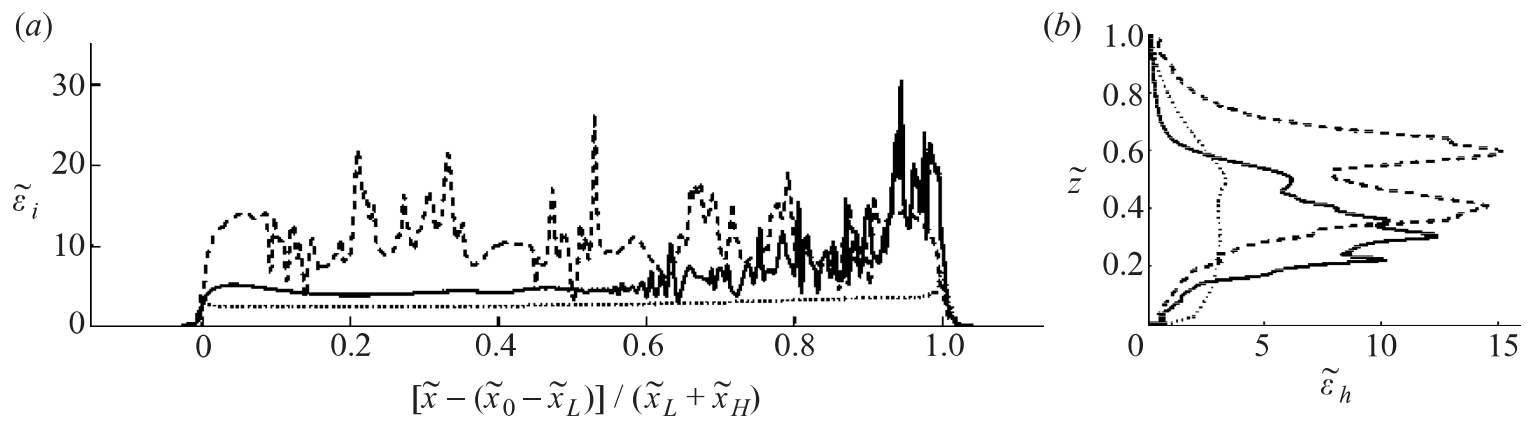

FIGURE 10. Dissipation at the interface of density currents $(R e=7071, \tilde{t}=9$, free-slip boundary conditions are imposed here). (a) Longitudinal distribution $\tilde{\varepsilon}_{i}$. (b) Vertical distribution $\tilde{\varepsilon}_{h}$. Dashed line, $\rho_{L} / \rho_{H}=0.99$; solid line, $\rho_{L} / \rho_{H}=0.2$; dotted line, $\rho_{L} / \rho_{H}=10^{-3}$. The streamwise coordinate has been rescaled so that the light and heavy fronts are located at 0 and 1 , respectively.

displaced closer to the heavy front with increasing density ratio (figure 10). Note that free-slip boundary conditions are used in figure 10, so that only the dissipation at the interface is considered here (the calculation of the dissipation is detailed in $\S 3.3$ ). Figure $10(a)$ shows that the maximum value of the dissipation at the head of the heavy front is larger at intermediate density contrast $\left(\rho_{L} / \rho_{H}=0.2\right)$ rather than at low and high density contrasts (see the peaks in figure $10 a$ at the abscissa corresponding to 0.95 dimensionless units approximately). These observations are likely to indicate that the dissipation at the interface subtly influences the shape and velocity of the head of the heavy current in the intermediate range of density ratios $0.1 \leqslant \rho_{L} / \rho_{H} \leqslant 0.6$, and accounts for the slight departure from theories.

Note that the maximum of the horizontally integrated vertical distribution of dissipation depicted in figure $10(b)$ decreases with increasing density contrast. Therefore, at higher density contrast $\left(0 \leqslant \rho_{L} / \rho_{H} \leqslant 0.1\right)$, the dissipation at the interface is expected to be insignificant and the computed heavy front velocities recover predictions from the shallow-water theories. Overall, the results obtained with free-slip boundaries are in fairly good agreement with the shallow-water theories and confirm their relevance provided wall friction can be neglected.

When wall friction is taken into account, the computed velocities are significantly lower than predictions from the inviscid theories. The discrepancy increases with increasing density contrast (see the triangles in figure 9b). Clearly, wall friction plays a significant role in the dynamics of highly contrasted density currents. Therefore, it must be taken into account to predict front velocities reliably. We derive in Appendix A a simple model that includes resistive effects for the prediction of the front velocity of density currents for arbitrary density contrasts. The analysis of Benjamin (1968) is extended to include a friction term modelled using a Chézy closure. Such an approach has already been used in the Boussinesq limit by Kranenburg (1978). Keeping in mind the simplicity and limitations of this approach, here we extend the analysis to cover the complete range of density contrast $0<\rho_{L} / \rho_{H}<1$. The velocities of the heavy and light fronts are then

$$
\begin{gathered}
\tilde{u}_{H}=\frac{1}{2} \sqrt{\frac{1}{1+\frac{1}{3} k \rho_{H} / \rho_{L}}} \sqrt{1+\frac{\rho_{H}}{\rho_{L}}}, \\
\tilde{u}_{L}=\frac{1}{2} \sqrt{\frac{1}{1+\frac{1}{3} k}} \sqrt{1+\frac{\rho_{L}}{\rho_{H}}},
\end{gathered}
$$


where $k$ is an energy loss coefficient (see Appendix A). From measurements of the front velocity in Boussinesq density currents, the energy loss coefficient was estimated experimentally to be $k \approx 0.6$ approximately (Kranenburg 1978; Bühler, Wright $\&$ Kim 1991).

Equations (3.2a) and (3.2b) are an extension of Benjamin's solutions (1.2a) and $(1.2 b)$ to the situation where resistive effects are significant. The value of the heavy front velocity $(3.2 a)$ remains finite even at high density contrast, in contrast to Benjamin's prediction $(1.2 a)$. The front velocities as computed in (3.2) with $k=0.6$ are plotted in figure 9 (dash-dotted line). Note that a $10 \%$ variation of $k$ around the value 0.6 leads to a $0.8 \%$ variation of the predicted light front velocity for the complete range of density ratio, and a $0.8 \%$ (resp. $5 \%$ ) variation of the predicted heavy front velocity of Boussinesq (resp. non-Boussinesq) density currents. Still, good agreement is found with currents spreading on a no-slip boundary for the complete range of density contrast. This simple model provides further evidence that resistive effects are responsible for the discrepancy between experimental data and inviscid theories.

We also compared the present results with the well-known Huppert \& Simpson's (1980) speed correction either for half-depth current or used in conjunction with the one-layer shallow-water model of Ungarish (2007). It was observed that for the light currents, the results given by models based on the Huppert \& Simpson's (HS) speed correction and $(3.2 b)$ are close to one another (the difference being less than $2 \%$ ). In contrast, for the heavy currents, the agreement between the computational results and the results with the models using the HS speed correction was observed to be reasonable only at moderate density ratio $\left(0.4 \leqslant \rho_{L} / \rho_{H}<1\right)$. For more contrasted density currents, the HS speed correction for half-depth current becomes singular and strongly overestimates the speed of the current. Conversely, the prediction of the current speed with the one-layer shallow-water model using the HS speed correction tends to be close to that of Ungarish's (2007) original model. For $\rho_{L} / \rho_{H} \rightarrow 0$, the model increasingly overestimates the current speed (the overestimate is about $40 \%$ for $\left.\rho_{L} / \rho_{H}=10^{-3}\right)$. This discrepancy may be partly due to the fact that the HS speed correction was obtained from measurements in the Boussinesq limit and its use at large density contrast may not be appropriate.

To assess the effect of the Reynolds number on the present results, we compared in figure 9 our numerical results obtained for $R e=5 \times 10^{4}$ (solid triangles) with that obtained for $R e=7071$ (open triangles). The front velocity of currents spreading on free-slip walls was observed to be independent of the Reynolds number. In contrast, the velocity of the currents spreading on the no-slip walls increases with the Reynolds number. For instance, the increase of front velocity of the light current between $R e=7071$ and $R e=5 \times 10^{4}$ is of $6 \%$ for the complete range of density ratio, whereas the increase of front velocity of the heavy current is of $6 \%$ in the Boussinesq limit and of $14 \%$ in the limit of large density contrast. This confirms the general trend that wall friction is a decreasing function of the Reynolds number (see e.g. Hager 1988; McKeon, Zagarola \& Smits 2005). We note that the value of $k=0.6$ used in figure 9 is an arbitrary choice. In fact, a similar trend of decreasing front velocity with increasing value of the energy loss coefficient $k$ in the present Chézy-corrected front speed law can be obtained (not shown here).

\subsection{Dissipation in the interface region and at rigid walls}

To further examine the role of wall friction, we determined the spatial distribution of dissipation in density currents. We first compute the dimensionless horizontally 

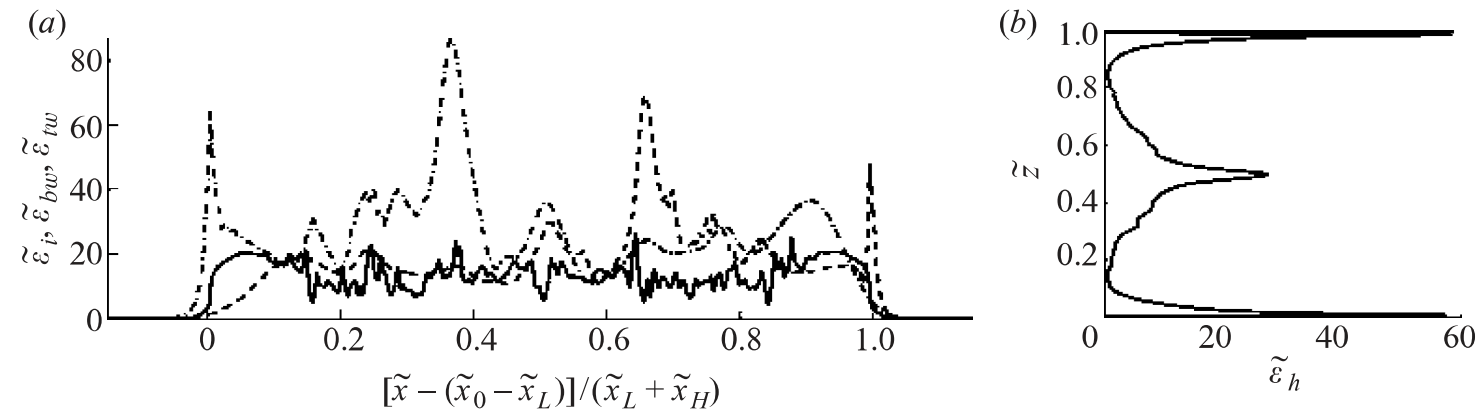

Figure 11. Dissipation in a Boussinesq density current $\left(\rho_{L} / \rho_{H}=0.99, R e=7071, \tilde{t}=9\right)$. (a) Longitudinal distribution. Solid line, $\tilde{\varepsilon}_{i}$ at the interface; dashed line, $\tilde{\varepsilon}_{b w}$ at the bottom wall; dashed-dotted line, $\tilde{\varepsilon}_{t w}$ at the top wall. $(b)$ Vertical distribution $\tilde{\varepsilon}_{h}$. The streamwise coordinate has been rescaled so that the light and heavy fronts are located at 0 and 1 , respectively.

integrated dissipation $\tilde{\varepsilon}_{h}(\tilde{z})$ as,

$$
\tilde{\varepsilon}_{h}(\tilde{z})=\frac{\varepsilon_{h} H}{\mu g A t}=\frac{1}{\tilde{L}} \int_{0}^{\tilde{L}} \tilde{S}_{i j} \tilde{S}_{i j} \mathrm{~d} \tilde{x},
$$

where the dimensional dissipation is normalized by $\mu g A t / H$, and $\tilde{S}_{i j}$ is the dimensionless strain rate tensor. Here, we wish to discriminate dissipation at the interface (indicated by the subscript $i$ ) from dissipation at the top and bottom walls (indicated by subscripts $t w$ and $b w$, respectively). We split the entire layer into approximately that associated with the lower wall $\left(\tilde{z} \leqslant \tilde{z}_{1}\right)$, with the interface $\left(\tilde{z}_{1}<\tilde{z}<\tilde{z}_{2}\right)$ and with the upper wall $\left(\tilde{z} \geqslant \tilde{z}_{2}\right)$. Here we identify $\tilde{z}_{1}$ and $\tilde{z}_{2}$ as the vertical locations of the absolute minima of $\tilde{\varepsilon}_{h}$ in the lower $\left(0 \leqslant \tilde{z}_{1} \leqslant 1 / 2\right)$ and upper $\left(1 / 2<\tilde{z}_{2} \leqslant 1\right)$ layers, respectively. We then compute the vertically integrated quantities $\tilde{\varepsilon}_{i}, \tilde{\varepsilon}_{t w}$ and $\tilde{\varepsilon}_{b w}$, namely

$$
\begin{gathered}
\tilde{\varepsilon}_{i}(\tilde{x})=\frac{1}{\tilde{z}_{2}-\tilde{z}_{1}} \int_{\tilde{z}_{1}}^{\tilde{z}_{2}} \tilde{S}_{i j} \tilde{S}_{i j} \mathrm{~d} \tilde{z}, \\
\tilde{\varepsilon}_{t w}(\tilde{x})=\frac{1}{1-\tilde{z}_{2}} \int_{\tilde{z}_{2}}^{1} \tilde{S}_{i j} \tilde{S}_{i j} \mathrm{~d} \tilde{z}, \\
\tilde{\varepsilon}_{b w}(\tilde{x})=\frac{1}{\tilde{z}_{1}} \int_{0}^{\tilde{z}_{1}} \tilde{S}_{i j} \tilde{S}_{i j} \mathrm{~d} \tilde{z} .
\end{gathered}
$$

We thus assume that for $\tilde{z} \leqslant \tilde{z}_{1}\left(\tilde{z} \geqslant \tilde{z}_{2}\right)$, the entire dissipation is due to the bottom (resp. upper) wall, whereas for $\tilde{z}_{1}<\tilde{z}<\tilde{z}_{2}$ it is due to the interface. In figures 11 and 12, we plot the spatial distribution of the dissipation inside a Boussinesq and a non-Boussinesq current, respectively.

The longitudinal and vertical distributions of the dissipation for the Boussinesq current are shown in figure 11. The flow for the case shown here $\left(\rho_{L} / \rho_{H}=0.99\right.$, $R e=7071)$ is highly chaotic and does not exhibit perfect top-bottom symmetry about $\tilde{y}=0.5$ or perfect left-right symmetry about $\tilde{x}_{0}$ (see figure $4 a$ ). Horizontal or vertical average does not totally erase asymmetry and accordingly the dissipation does not exhibit perfect symmetry. An ensemble average over repeated realizations (with different random disturbances and as a result different evolution of interfacial roll-up) would lead to perfect symmetry. The vertical distribution of $\tilde{\varepsilon}_{h}$ shown in figure $11(b)$ reveals three local maxima. The one at the centre of the channel corresponds to the average location of the interface, while the other two are located at the walls. 

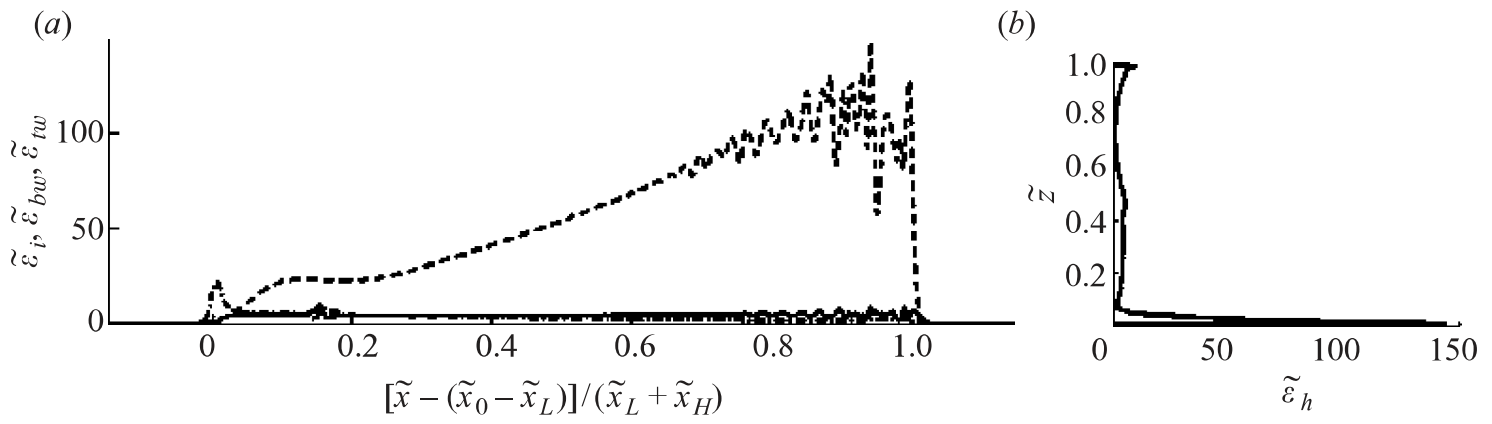

Figure 12. Same as figure 11 for a non-Boussinesq density current $\left(\rho_{L} / \rho_{H}=10^{-3}\right)$.

Hence, they capture the effect of the enhanced dissipation at the boundaries. The peak value of dissipation at both walls is about twice as large as at the interface. However, the dissipation is narrowly peaked at the walls while it is more broadly distributed at the interface. From figure $11(b)$ it can be seen that well-defined minima of $\tilde{\varepsilon}_{h}$ exist in both the upper and lower layers, thus enabling an unambiguous identification of the separation points, $\tilde{z}_{1}$ and $\tilde{z}_{2}$, between the wall and interfacial layers.

The magnitude of the longitudinal distribution of vertically averaged dissipations, $\tilde{\varepsilon}_{i}, \tilde{\varepsilon}_{t w}$ and $\tilde{\varepsilon}_{b w}$, in the Boussinesq current are of the same order of magnitude (figure 11a). Although the precise value of $\tilde{\varepsilon}_{i}, \tilde{\varepsilon}_{t w}$ and $\tilde{\varepsilon}_{b w}$ depends on how $\tilde{z}_{1}$ and $\tilde{z}_{2}$ are located, the results to be inferred are insensitive to their precise location. At the interface, dissipation is nearly uniform along the body of the current, while dissipation at the walls exhibits strong variations, which are due to the influence of the interfacial vortex structures on the near-wall shear layers. Especially, near the heavy and light front locations, the peaks of $\tilde{\varepsilon}_{t w}$ and $\tilde{\varepsilon}_{b w}$ are found to correspond to the location of the vortex structures at the interface (see figure 4).

At high density contrast, the distribution is dramatically different, as shown in figure 12. Dissipation at the bottom wall is larger than dissipation at the interface and at the top wall by one order of magnitude. For instance, at the instant shown in figure 12, the peak dissipations at the bottom wall, at the interface and at the top wall are 145,5 and 10 , respectively. Although these values fluctuate and evolve over time, the relative importance remains the same. Note also that the dissipation at the interface is significantly lower than that in figure 11 (the dimensionless values are 5 for the nonBoussinesq current $v$ s. 25 for the Boussinesq current). From the location of the initial lock, the dissipation at the bottom wall linearly increases and reaches a maximum close to the heavy front. It thus appears that the heavy front is strongly influenced by wall friction, contributing to the increasing departure of the heavy front velocity at high density contrast from that predicted by shallow-water theories that do not include wall effects. In contrast, even at high density ratios, the dissipation at the top boundary remains of the same amplitude as at small density ratio and thus Benjamin's dissipationless model is able to capture the velocity of the light front quite well.

\section{Intermediate Reynolds numbers}

\subsection{Shape of density currents}

Figure 13 shows the shape of the current for three different density contrasts, namely $\rho_{L} / \rho_{H}=10^{-3}, 0.4$, and 0.99 at three low-to-moderate Reynolds number $R e=71,224$ and 707, respectively. In all cases, roll-up of the interface and formation of coherent vortex structures is not observed. The destabilizing effect of the interfacial shear 
(a)

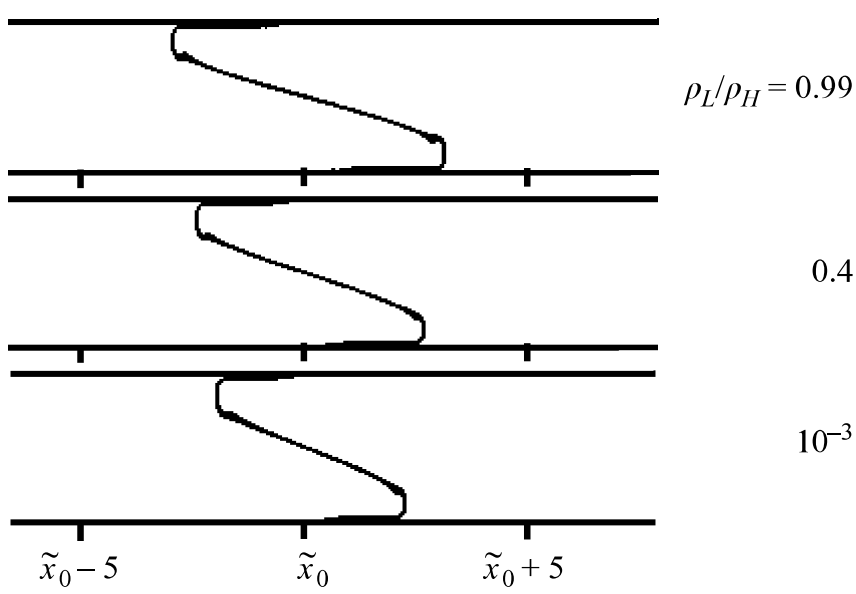

(b)

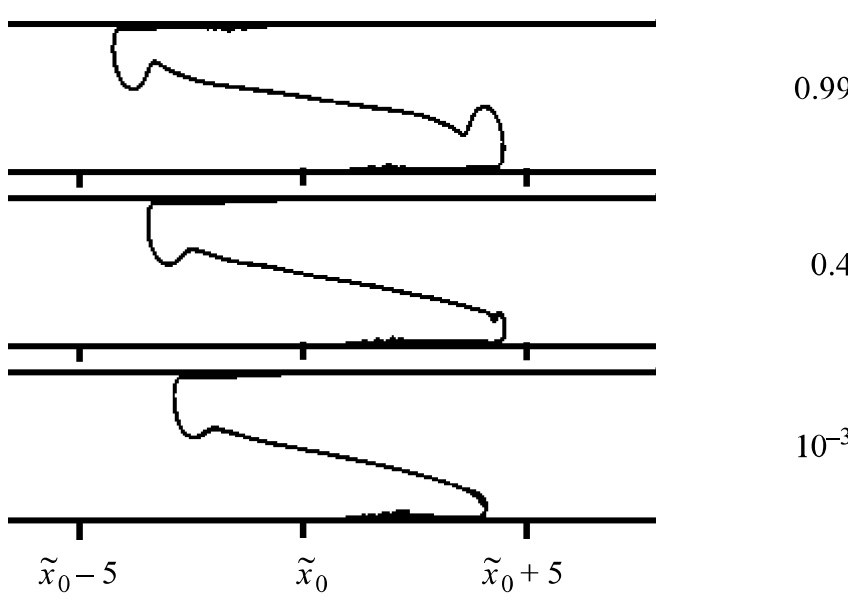

(c)

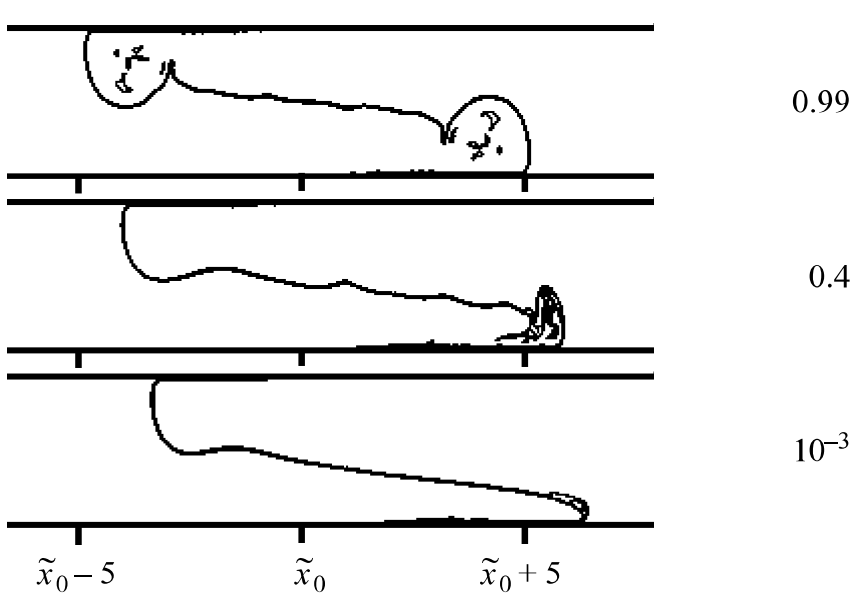

FIGURE 13. Iso-contours of $\tilde{\rho}$ at $\tilde{t}=9$. Results for $(a) R e=71,(b) R e=224$ and $(c) R e=707$. Values of plotted iso-contours are $\tilde{\rho}=0.05-0.275-0.5-0.725-0.95$.

is not strong enough to overcome the stabilizing effect of stratification (Drazin \& Reid 1981; Bonometti \& Balachandar 2008). At low density contrast $\rho_{L} / \rho_{H}=0.99$, a depression separating the heavy and light heads of the current from the body is visible. This depression is clearly seen at $R e=224$ and 707. It is much weaker in size at $R e=71$, but can be observed upon careful inspection. With increasing density contrast, the depression at the light front becomes progressively weaker, while that at the heavy front shifts toward the front of the current and eventually disappears. 
Also with increasing density contrast, the shape of the heavy front becomes more slender, while the head of the light front becomes more rounded. These observations are consistent with those of available experimental results (Lowe et al. 2005) and computations (Birman et al. 2005; Etienne et al. 2005) performed at higher Reynolds number.

\subsection{Front velocity}

The temporal evolution of the heavy and light front velocities is depicted in figure 14 for $R e=71,224$ and 707 and $\rho_{L} / \rho_{H}=10^{-3}, 0.4$ and 0.99 , respectively. As illustrated in figure 8 , with increasing density contrast, the gap between light and heavy front velocities increases. However, at lower $R e$, the velocity of the heavy front decreases faster than that of the light front and the gap diminishes and reverses accordingly. For $R e=71$, the velocities of the light and heavy front become nearly equal for $\tilde{t} \geqslant 15$ for all density ratios. Note in figure 13 that at this value of $R e$, the shape of the currents is almost symmetric.

At the highest Reynolds numbers considered in this study (see for instance figure 8 for $R e=7071$ ), the velocity of the heavy (light) front was observed to increase (decrease) with density contrast and remain so over the entire duration of computation. The situation at low Reynolds number shown in figure 14 is more complex. For $R e=707$, the only anomaly seems to be the behaviour of the velocity of the heavy front for the largest density contrast. Although it reaches a larger peak value around $\tilde{t} \approx 1.75$, it does not exhibit any period of nearly constant value, but continues to decrease. In fact, it eventually becomes smaller than the speed of the heavy front at lower density ratios. At even lower Reynolds number $(R e=224)$, the light fronts exhibit a brief period of constant velocity, and for $\tilde{t}>10$, undergo a slow decay. The velocity of the heavy front shows a rapid decay after it reaches the peak. Quickly, the heavy front begins to propagate more slowly than the light front. This trend continues and amplifies at the lowest Reynolds number $(R e=71)$ considered.

The reason for this is the competition between inertia and wall friction. On the one hand, increasing the density contrast obviously increases the inertia of the heavy fluid through the buoyancy force which is likely to increase the front velocity; on the other hand, increasing the density contrast leads to an increase of the friction between the heavy fluid and the bottom wall which is likely to decrease the front velocity. At high $R e$, inertia is dominant and the velocity is augmented, whereas at low $R e$, friction is dominant and the velocity is diminished compared to that of Boussinesq currents.

An indirect confirmation is provided when we compute density currents with the same flow parameters but with free-slip boundaries. In that case, the wall friction is nullified and it is possible to isolate the effects of inertia. Figure 15 shows density currents which have the same properties as those of figure $13(b)$ i.e. $R e=224$ and $\rho_{L} / \rho_{H}=0.99,0.4,10^{-3}$, respectively, but obey free-slip boundary conditions. We recall that the currents of figure 13(b) were computed with no-slip boundary conditions. Clearly, the heavy front spreads faster at high density contrast, unlike the heavy fronts of figure 13(b). This confirms that wall friction is responsible for the reversing of the hierarchy of front velocities between slightly and highly contrasted currents.

For a prescribed density contrast, the transient time of the heavy (resp. light) current, measured as the dimensionless time for which the current reaches its maximum velocity, increases (resp. decreases) when increasing $R e$ (figure 14). This tendency has already been observed in the situation where the density contrast is increased at a fixed $R e$, as discussed in $\S 3.2 .3$, and the reason for this is of the same nature. 

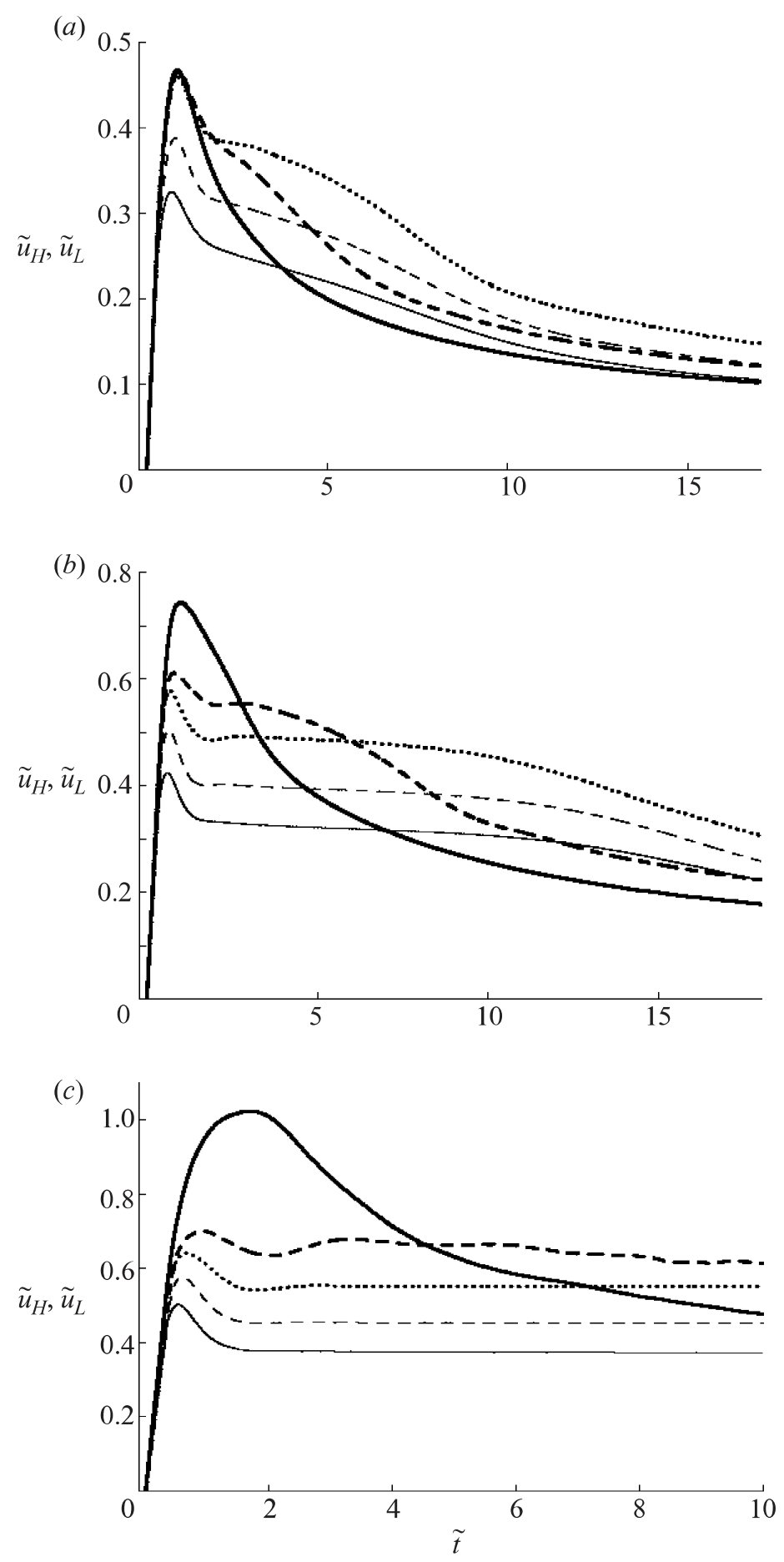

FIGURE 14. Time evolution of the heavy front velocity $\tilde{u}_{H}$ (thick line) and light front velocity $\tilde{u}_{L}$ (thin line). The dotted, dashed and solid lines correspond to $\rho_{L} / \rho_{H}=0.99,0.4$ and $10^{-3}$, respectively. (a) $R e=71,(b) R e=224$ and (c) $R e=707$.

\section{Summary}

We carried out a numerical investigation of nearly immiscible density currents with various density contrasts and Reynolds numbers. The goal was to shed light on the role of wall friction on the dynamics of these flows. For this purpose, we computed two-dimensional lock-exchange flows using a finite-volume interface capturing method that allows us to explore a wide range of density contrast and Reynolds number, $10^{-3} \leqslant \rho_{L} / \rho_{H} \leqslant 0.99$ and $70 \leqslant R e \leqslant 5 \times 10^{4}$, respectively. 


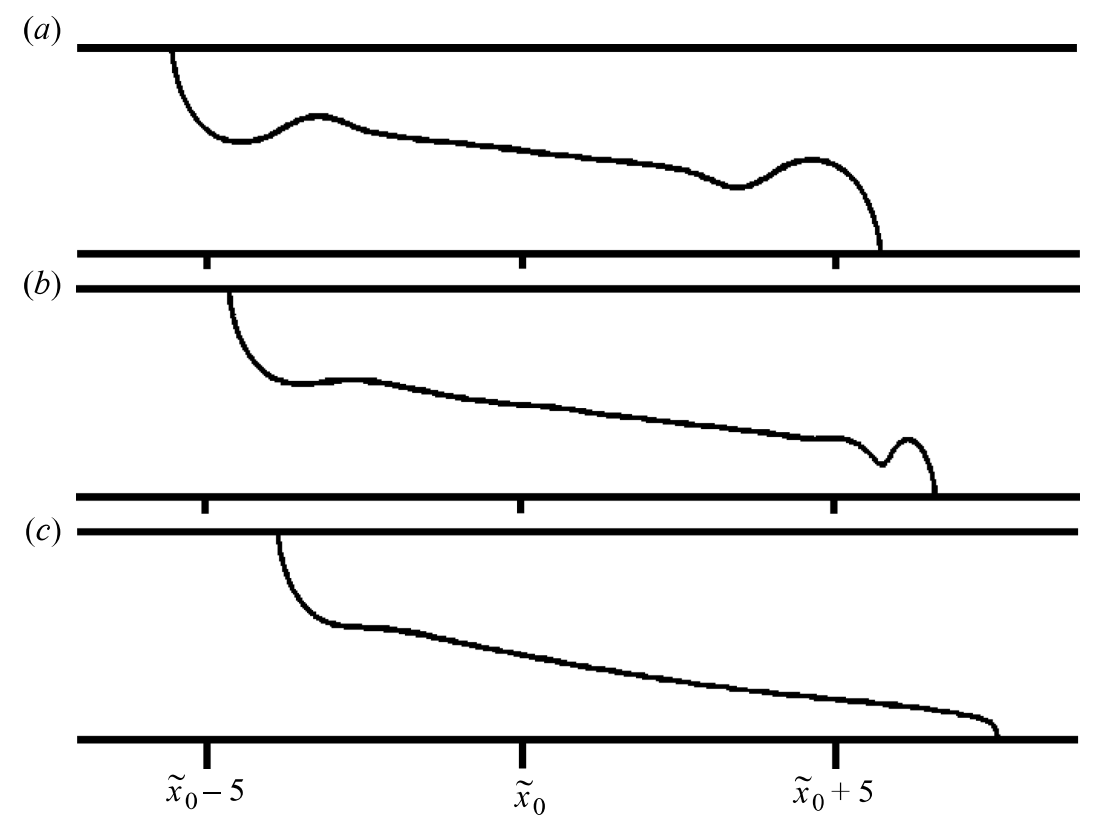

FiguRE 15. Iso-contours of $\tilde{\rho}$ at $\tilde{t}=9(R e=224)$. Free-slip boundary conditions are imposed. (a) $\rho_{L} / \rho_{H}=0.99,(b) \rho_{L} / \rho_{H}=0.4,(c) \rho_{L} / \rho_{H}=10^{-3}$. Values of plotted iso-contours are $\tilde{\rho}=$ $0.05-0.275-0.5-0.725-0.95$.

By imposing either free-slip or no-slip boundary conditions, we were able to discriminate the contribution of dissipation at the interface and at boundaries. When wall friction is neglected (by imposing free-slip conditions), the light front velocity of density currents is described well by Benjamin's half-depth dissipationless speed result (within $0.5 \%$ ) for the complete range of density ratio. This supports the view that in the Boussinesq limit, the effect of large-scale vortical structures and mixing at the interface on the speed of propagation of the currents is negligibly small. The heavy front velocity is fairly well predicted by the one- and two-layer shallow-water theories. The agreement is fairly good for density ratios close to 0 or 1 , as dissipation in the interface region does not affect the head of the current. At intermediate density contrast, however, the dissipation in the interface region becomes significant in the vicinity of the head of the current. This possibly influences the shape of the head and slightly decreases the front velocity which in turn exhibits small oscillations. Overall, this provides further evidence that the theoretical models based on the inviscid shallow-water approximations and Benjmain's steady state result can fairly well reproduce the front velocity of the light and heavy currents for the complete range of density ratios, provided wall effects can be neglected.

However, except for a current of small density contrast, the effects of wall friction modify the velocity of the current. As soon as wall friction is taken into account (by imposing no-slip conditions), the front velocity of the light and heavy fluids is lower than predicted by inviscid theories. At high density contrast, most of the dissipation is observed to take place at the bottom wall and near the heavy front. A simple model taking into account wall friction (see Appendix A) is proposed for the prediction of density currents spreading on rigid boundaries. Good agreement is found with present numerical results providing further evidence that wall effects are the crucial ingredient for an accurate prediction of the front velocity of highly contrasted density currents.

In this work, we assumed that the two fluids have identical and constant dynamic viscosities. However, the present results (Appendix B) along with those of Birman 
(a) $D$

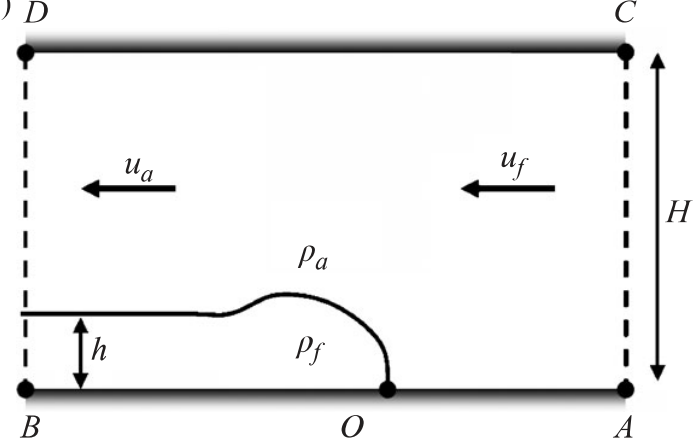

(b) $A$

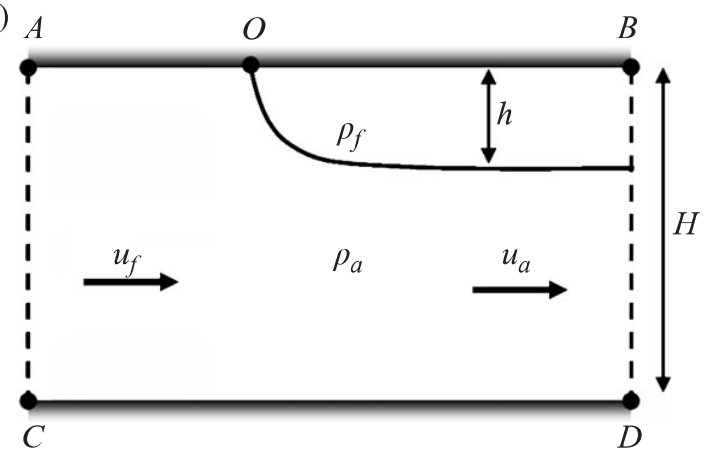

FIGURE 16. Schematic diagram of an idealized $(a)$ heavy $(b)$ light gravity current in the rest frame of reference of the current.

et al. (2005) show that the dynamics of density currents is mostly independent of the assumption of constant dynamic or kinematic viscosity for the whole range of density ratio when the Reynolds number, based on the heavier fluid, is large i.e. $R e \geqslant O\left(10^{3}\right)$.

We also investigated the dynamics of non-Boussinesq density currents at moderate Reynolds number. In contrast to high-Re configurations, no vortical structure is observed at the interface. However, present results reveal a more complex behaviour than for high- $R e$ currents. The main reason for this is the competition between inertia and wall friction effects that both increase at low-to-moderate Reynolds number, especially for high density contrasts.

This work focused on the initial slumping phase of non-Boussinesq density currents in the lock-exchange configuration. It could be extended in many ways. First, the analysis of the slumping motion of non-Boussinesq currents with arbitrary initial depth ratio is required in order to test the accuracy of inviscid theories in the case of small depth releases, especially at moderate-to-high density contrasts. It is also of particular interest to explore the effect of the density ratio on the self-similar inertial and viscous regimes that occur immediately after the slumping phase, in particular when the initial volume of the intruding fluid is small compared to that of the ambient fluid. These directions are left for future work.

The authors would like to thank the referees for their valuable and insightful comments, which have led to a significant improvement of the original version of the paper. This work benefited from the NSF grant EAR-0609712.

\section{Appendix A. Derivation of the model (3.2): revisiting Benjamin's analysis}

In this Appendix, we present the derivation of (3.2) used in the prediction of the front velocity of density currents propagating in between no-slip boundaries, at arbitrary density contrasts. Following Benjamin's analysis, we consider the control volume including the head of a gravity current of density $\rho_{f}$ and height $h$ propagating in an ambient fluid of density $\rho_{a}$ in a channel of height $H$ (figure 16). The fluid constituting the gravity current can be either heavy or light, therefore $\rho_{f}$ can be $\rho_{H}$ or $\rho_{L}$ and correspondingly $\rho_{a}$ can be $\rho_{L}$ or $\rho_{H}$. The size of the control volume is chosen to be $O\left(H^{2}\right)$. In a reference frame moving with the current head, the velocity far upstream and downstream are denoted by $u_{f}$ and $u_{a}$ (figure 16). The conservation of mass is,

$$
u_{f} H=u_{a}(H-h) .
$$


Conservation of the horizontal component of the momentum flux may then be written as

$$
\begin{aligned}
\int_{A}^{C} \rho u^{2} \mathrm{~d} z-\int_{B}^{D} \rho u^{2} \mathrm{~d} z & =\int_{B}^{D} p \mathrm{~d} z-\int_{A}^{C} p \mathrm{~d} z \\
& +\int_{A}^{C} \tau_{x x} \mathrm{~d} z-\int_{B}^{D} \tau_{x x} \mathrm{~d} z+\int_{D}^{C} \tau_{x z} \mathrm{~d} x-\int_{B}^{A} \tau_{x z} \mathrm{~d} x,
\end{aligned}
$$

where $\tau_{i j}$ is the stress tensor. The normal stress along the vertical boundaries $A C$ and $B D$ is assumed to be smaller than the shear stress along the horizontal boundaries $A B$ and $C D$ of the control volume, so the third and fourth terms in the right-hand side of (A 2) can be neglected. We further assume that the shear stress at the surface in contact with the intruding fluid is much larger than that at the opposite surface. Hence, the last term of the right-hand side of (A 2) is also neglected. There are several approaches to relate the boundary shear stress to the height, velocity and properties of the fluid (see Hogg \& Pritchard 2004 for a discussion). Here, we adopt a common and simple model by using a semi-empirical Chézy-type law closure, defined as

$$
\tau_{x z} \equiv \tau_{b}=-\frac{1}{2} k \max \left(\rho_{a}, \rho_{f}\right) u_{f}^{2},
$$

where $k$ is an energy loss coefficient. Assuming the pressure to be hydrostatic on $B D$ and $A C$, and using the velocity distributions in the reference frame moving with the current head, the momentum balance (A 2) becomes

$$
\frac{1}{2}\left(\rho_{f}-\rho_{a}\right) g h(h-2 H)=\left(p_{A}-p_{B}\right) H-\rho_{a} u_{a}^{2}(H-h)+\rho_{a} u_{f}^{2} H+\tau_{b} H .
$$

We now apply Bernoulli's equation along $A B$ to eliminate the pressure term. This gives

$$
p_{B}=p_{A}+\frac{1}{2} \rho_{a} u_{f}^{2}
$$

Finally, substituting (A 3) and (A 5) into (A 4) and using the continuity equation (A 1), we obtain the following expression for the front velocity of the propagating current,

$$
u_{f}=\sqrt{\frac{g\left|\rho_{f}-\rho_{a}\right|}{\rho_{a}} \frac{h(2 H-h)}{H\left(\frac{H+h}{H-h}+k \frac{\max \left(\rho_{f}, \rho_{a}\right)}{\rho_{a}}\right)}} .
$$

In dimensionless variables this becomes

$$
\tilde{u}_{f}=\sqrt{\frac{\tilde{h}(2-\tilde{h})}{\frac{1+\tilde{h}}{1-\tilde{h}}+k \frac{\max \left(\rho_{f}, \rho_{a}\right)}{\rho_{a}}}} \sqrt{1+\frac{\rho_{f}}{\rho_{a}}} .
$$

In the case of lock-exchange flows, substituting $\tilde{h}=1 / 2$ in (A 7) yields (3.2). Here, it must be cautioned that the present closure for the height of the propagating front is an arbitrary choice. This assumption is a good approximation for the light current height within the complete range of density ratio and the heavy current height at moderate density ratio. However, the main limitation lies in the range of high density ratio for which the height of the heavy current is smaller. This limitation may be improved by using a more advanced closure which would express the height of the propagating heavy front as a function of the density ratio $\rho_{H} / \rho_{L}$, based on results of shallow-water approximations for instance. However, to keep the simplicity of the model, the present closure is applied for the whole range of density ratio. 


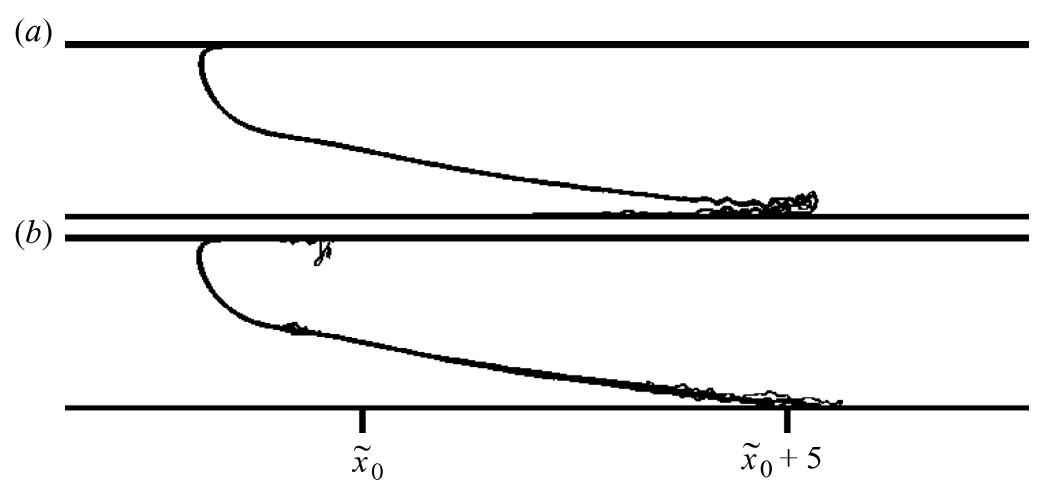

FIGURE 17. Iso-contours of $\tilde{\rho}$ at $\tilde{t}=4.2\left(\rho_{L} / \rho_{H}=10^{-3}, R e=7071\right)$ with two different assumptions concerning the viscosities. $(a) \mu_{H}=\mu_{L}$ and $(b) v_{H}=v_{L}$. Values of plotted iso-contours are $\tilde{\rho}=0.05-0.275-0.5-0.725-0.95$.

\section{Appendix B. On the assumption of constant viscosity}

In this Appendix, we consider the effect of the assumption of constant viscosity. As mentioned in $\S 2$, we assumed that both fluids have the same dynamic viscosity instead of the same kinematic viscosity. These two assumptions are equivalent in the Boussinesq case, but their influence is non-trivial at larger density contrasts. In the range $3 \times 10^{3} \leqslant R e \leqslant 10^{4}$, Birman et al. (2005) observed that the results for $S c=1$ currents of moderate density contrast are qualitatively independent of whether the dynamic or the kinematic viscosity of the heavy and light fluids are the same. As shown in figure 17, this conclusion can be extended to the case of high-Sc currents of high density contrast as well. For instance, at $R e=7071$ and $\rho_{L} / \rho_{H}=10^{-3}$, we found that the temporal evolution of the front position is the same to within $2 \%$ during the entire duration of the computation.

The shapes of the currents are similar as well, except at the head of the heavy current which is slightly lifted up in the case $\mu_{H}=\mu_{L}$. This can be explained by comparing the value of the light fluid viscosity in both cases, say $\mu_{L}^{(a)}$ and $\mu_{L}^{(b)}$. As the viscosity of the heavy fluid $v_{H}$ is imposed by the Reynolds number through (2.8), we can express the viscosity of the light fluid in terms of $v_{H}$ as $\mu_{L}^{(a)}=\mu_{H}=\rho_{H} v_{H}$ and $\mu_{L}^{(b)}=\rho_{L} v_{L}=\rho_{L} v_{H}$. So the ratio between the viscosity of the light fluid in the two cases is $\mu_{L}^{(a)} / \mu_{L}^{(b)}=\rho_{H} / \rho_{L}=10^{3}$. Thus, for the large-density-ratio case, the light fluid is more viscous by three orders of magnitude when the dynamic viscosities are the same $\left(\mu_{H}=\mu_{L}\right)$ than when the kinematic viscosities are equal $\left(v_{H}=v_{L}\right)$. In the former case, the interstitial film of light fluid between the head of the heavy current and the bottom wall is thicker and makes the head of the heavy current lift up.

Etienne et al. (2005) also performed analogous simulations by varying the viscosity model (same dynamic viscosity vs. same kinematic viscosity). In contrast with the present conclusion, they observed the front velocity to be somewhat sensitive to the corresponding assumption. The reason for this contradiction lies in the choice of the characteristic viscosity in the definition of the Reynolds number. In the present work, the Reynolds number is defined based on the viscosity of the heavier fluid, while Etienne et al. (2005) define $R e$ based on the viscosity of the lighter fluid. As a result, in the present simulations, at a fixed Reynolds number, only the light fluid is sensitive to the viscosity assumption while the viscosity of the heavy fluid remains the same. The situation is opposite in the work of Etienne et al. (2005). Consequently, the viscosity of the heavy fluid is proportional to the density contrast $\rho_{H} / \rho_{L}$, thus leading to very different situations at high density contrast. For comparison, we give in table 4 


$$
\begin{aligned}
& \begin{array}{cccc} 
& (a) & (b) & (c) \\
\rho_{L} / \rho_{H} & R e=\sqrt{g \frac{\Delta \rho}{\rho_{L}+\rho_{H}} H} H / v_{H} & R e_{B}=\sqrt{g \frac{\Delta \rho}{\rho_{H}} H} H / v_{H} & R e_{E}=\sqrt{g \frac{\Delta \rho}{\rho_{L}} \frac{H}{2}} \frac{H}{2} / \nu_{L} \\
& \text { (Assumption: } \mu_{L}=\mu_{H} \text { ) } & \text { (Assumption: } v_{L}=v_{H} \text { ) } & \text { (Assumption: } \mu_{L}=\mu_{H} \text { ) (Assumption: }
\end{array} \\
& \begin{array}{cclll}
0.99 & 7071 & \approx 10^{4} & \approx 3500 & \approx 3500 \\
0.99 & 5 \times 10^{4} & \approx 7 \times 10^{4} & \approx 2.5 \times 10^{4} & \approx 2.5 \times 10^{4} \\
10^{3} & 7071 & \approx 7071 & \approx 80 & \approx 8 \times 10^{5} \\
10^{3} & 5 \times 10^{4} & \approx 5 \times 10^{4} & \approx 560 & \approx 5.6 \times 10^{6}
\end{array}
\end{aligned}
$$

TABLE 4. Comparison of the value of the Reynolds number as defined in: $(a)$ the present work; (b) Birman et al. (2005); (c) Etienne et al. (2005).

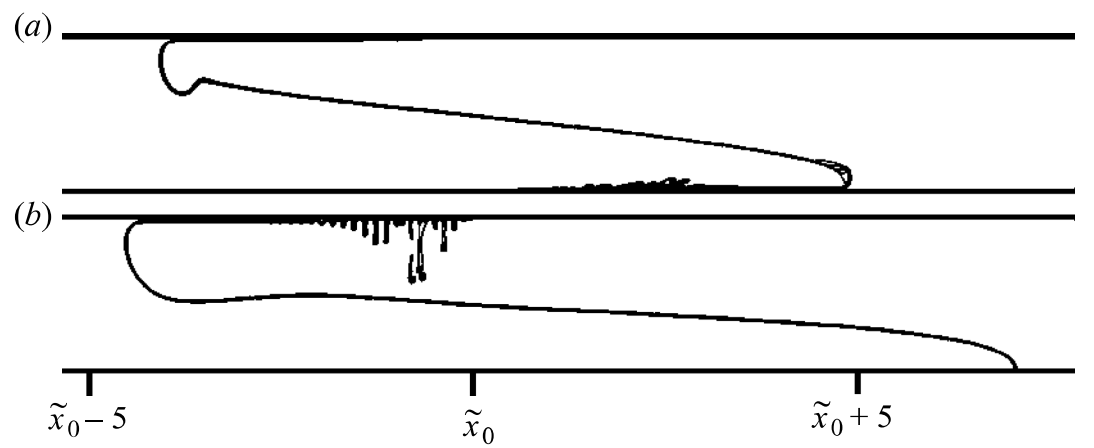

FiguRE 18. Iso-contours of $\tilde{\rho}$ at $\tilde{t}=12.7\left(\rho_{L} / \rho_{H}=10^{-3}, R e=224\right)$ for $(a) \mu_{H}=\mu_{L}$ and

(b) $v_{H}=v_{L}$. Values of plotted iso-contours are $\tilde{\rho}=0.05-0.275-0.5-0.725-0.95$.

the value of $R e$ for two different density ratios at the two highest Reynolds numbers investigated here, with the scaling used in the present work, as well as in Birman et al. (2005) and Etienne et al. (2005) $\left(R e, R e_{B}\right.$ and $R e_{E}$ are the Reynolds numbers with the present scaling, Birman's scaling and Etienne's scaling, respectively).

Although in figure 17, only results for a single case of high density contrast at $R e=7071$ are presented, the behaviour remains the same at other density contrasts and Reynolds numbers. The present results along with those of Birman et al. (2005) show that the dynamics of density currents are mostly independent of the assumption of constant dynamic or kinematic viscosity for the whole range of density ratios when the Reynolds number, based on the heavier fluid, is large, i.e. $R e \geqslant O\left(10^{3}\right)$. Further tests with different viscosity ratios $\mu_{H} / \mu_{L}$ ranging from 1 to $10^{2}$ were also performed and suggest that this result holds even when the viscosity ratio is not unity.

However, the rate of spreading of density currents becomes dependent on the assumption on constant dynamic or kinematic viscosity when viscous effects are significant, as illustrated in figure 18 showing the shape of currents with $R e=224$. Here, the shape and position of both the heavy and light fronts are strongly different depending on whether we choose $\mu_{H}=\mu_{L}$ or $v_{H}=v_{L}$. The topology of the currents shown in figure 18 is at the same dimensionless time. We verified that the conclusion is the same when we consider the same displaced volume rather than the same time. As the current spreads, the velocity difference becomes about $10 \%$ and $30 \%$ for the light and heavy fronts, respectively.

Owing to the no-slip condition at the walls, an unstable layer of heavy fluid has developed at the upper wall (figure 18b) and viscous fingers falling from the top wall are observed. On the contrary, no finger is observed in case $(a)$. This difference 
is again related to the viscosity contrast between the two fluids. In the absence of capillary effects, any heavy layer of fluid located above a lighter fluid is unstable. Therefore, the layers of heavy fluid in both figures $18(a)$ and $18(b)$ are unstable. As the maximum velocity of the viscous digitations is proportional to the inverse of the ambient fluid viscosity $1 / \mu_{L}$ (Saffman \& Taylor 1958), the velocity of the fingers in case $(b)$ is three orders of magnitude larger than in case $(a)$. Note that the sedimentation velocity of a spherical drop in a viscous liquid obeys the same scaling law. Overall, this qualitatively explains why viscous digitations are observed only in case $(b)$.

\section{REFERENCES}

Baines, W. D., Rottman, J. W. \& Simpson, J. E. 1985 The motion of constant-volume air cavities released in long horizontal tubes. J. Fluid Mech. 161, 313-327.

Benjamin, T. B. 1968 Density currents and related phenomena. J. Fluid Mech. 31, 209-248.

Birman, V., Martin, J. E. \& Meiburg, E. 2005 The non-Boussinesq lock-exchange problem. Part 2. High-resolution simulations. J. Fluid Mech. 537, 125-144.

BonometTi, T. \& BalachandaR, S. 2008 Effect of Schmidt number on the structure and propagation of density currents. Theor. Comput. Fluid. Dyn. 22, 341-361.

Bonometti, T. \& Magnaudet, J. 2006 Transition from spherical cap to toroidal bubbles. Phys. Fluids 18, 052102.

BonometTi, T. \& Magnaudet, J. 2007 An interface-capturing method for incompressible two-phase flows. Validation and application to bubble dynamics. Intl J. Multiphase Flow 33, 109-133.

BoRIs, J. P. \& Book, D. L. 1973 Flux-corrected transport : I. SHASTA, a fluid transport algorithm that works. J. Comput. Phys. 18, 248-283.

BRitTeR, R. \& Linden, P. 1980 The motion of a front of a gravity current travelling down an incline. J. Fluid Mech. 99, 531-543.

BüHLER, J., Wright, S. J. \& KIM, Y. 1991 Gravity currents advancing into a coflowing fluid. J. Hydraul. Res. 29, 243-257.

Cantero, M., Balachandar, S., Garcia, M. \& Ferry, J. 2006 Direct numerical simulations of planar and cyindrical density currents. J. Appl. Mech. 73, 923-930.

Cantero, M. I., Lee, J. R., Balachandar, S. \& Garcia, M. H. 2007 On the front velocity of gravity currents. J. Fluid Mech. 586, 1-39.

Daly, B. \& Pracht, W. 1968 Numerical study of density-current surges. Phys. Fluids 11, 15-30.

Drazin, P. G. \& ReID, W. H. 1981 Hydrodynamic Stability, 2nd edn. Cambridge University Press.

Dressler, R. F. 1952 Hydraulic resistance effect upon the dam-break functions. J. Res. Natl. Bur. Stand. 49, 217-225.

Dressler, R. F. 1954 Comparison of theories and experiments for the hydraulic dam-break wave. In Publication 38 de l'Association Internationale d'Hydrologie, pp. 319-328.

ERManYuK, E. V. \& GaVRILOV, N. V. 2007 A note on the propagation speed of a weakly dissipative gravity current. J. Fluid Mech. 574, 393-403.

Etienne, J., Hopfinger, E. J. \& Saramito, P. 2005 Numerical simulations of high density ratio lock-exchange flows. Phys. Fluids 17, 036601.

Gardner, G. C. \& Crow, I. G. 1970 The motion of large bubbles in horizontal channels. J. Fluid Mech. 43, 247-255.

Grant, G. B., Jagger, S. F. \& Lea, C. J. 1998 Fires in tunnels. Phil. Trans. R. Soc. Lond. A 356, 2873-296.

Gröbelbauer, H. P., Fanneløp, T. K. \& Britter, R. E. 1993 The propagation of intrusion fronts of high density ratio. J. Fluid Mech. 250, 669-687.

HaGer, W. H. 1988 Abflussformen für turbulente Strömungen. Wasserwirtschaft 78, 79-84.

Härtel, C., Meiburg, E. \& Necker, F. 2000 Analysis and direct numerical simulation of the flow at a gravity-current head. Part 1. Flow topology and front speed for slip and no-slip boundaries. J. Fluid Mech. 418, 189-212.

HogG, A. J. \& PritchaRd, D. 2004 The effects of hydraulic resistance on dam-break and other shallow inertial flows. J. Fluid Mech. 501, 179-212. 
Hoult, D. 1972 Oil spreading in the sea. Annu. Rev. Fluid Mech. 4, 341-368.

Huppert, H. E. \& Simpson, J. E. 1980 The slumping of density currents. J. Fluid Mech. 99, 785-799. von KÁrmán, T. 1940 The engineer grapples with nonlinear problems. Bull. Am. Math. Soc. 46, 615-683.

Keller, J. J. \& Chyou, Y. P. 1991 On the hydraulic lock exchange problem. J. Appl. Maths Phys. 42, 874-909.

Klemp, J. B., Rotunno, R. \& Skamarock, W. C. 1994 On the dynamics of density currents in a channel. J. Fluid Mech. 269, 169-198.

Kranenburg, C. 1978 Internal fronts in two-layer flow. ASCE J. Hydaul. Div. 104, HY10, 1449-1453.

LAMB, H. 1945 Hydrodynamics, 6th edn. Cambridge University Press.

LAUber, G. \& HaGeR, W. H. 1998 Experiments to dam-break wave: horizontal channel. J. Hydraul. Res. 36, 291-307.

Lowe, R. J., Rottman, J. W. \& Linden, P. F. 2005 The non-Boussinesq lock-exchange problem. Part 1. Theory and experiments. J. Fluid Mech. 537, 101-124.

McKeon, B. J., Zagarola, M. V. \& Smits, A. J. 2005 A new friction factor relationship for fully developed pipe flow. J. Fluid Mech. 538, 429-443.

Marino, B., Thomas, L. \& Linden, P. 2005 The front condition for density currents. J. Fluid Mech. 536, 49-78.

Martin, J. C. \& Moyce, W. J. 1952 An experimental study of the collapse of liquid columns on a rigid horizontal plane. Phil. Trans. R. Soc. A 244, 312-324.

Necker, F., Härtel, C., Kleiser, L. \& Meiburg, E. 2005 Mixing and dissipation in particle-driven density currents. J. Fluid Mech. 545, 339-372.

Ozgökmen, T., Fischer, P., Duan, J. \& Iliescu, T. 2004 Three-dimensional turbulent bottom density currents from a high-order nonhydrostatic spectral element model. J. Phys. Oceanogr. 34, 2006-2026.

Ritter, A. 1892 Die fortplanzung der wasserwellen. Z. Verein. Deutsch. Ing. 36, 947-954.

RotTman, J. \& Simpson, J. 1983 Density currents produced by instantaneous releases of a heavy fluid in a rectangular channel. J. Fluid Mech. 135, 95-110.

SAfFman, P. G. \& TAYLOR G. I. 1958 The penetration of a fluid into a porous medium or Hele-Shaw cell containing a more viscous liquid. Proc R. Soc. Lon. A 245, 312-329.

DE SAINT-VenANT, B. 1871 Théorie du movement non permanent des eaux, avec application aux crues des rivières et à l'introduction des marées dans leur lit. C. R. Acad. Sci. 73, 147-154, 237-340.

SchmidT, W. 1911 Zur Mechanik der boen. Z. Met. 28, 355-362.

SchoкLitsch, A. 1917 Uber Dammbruchwellen. Sitzungsber. Akad. Wissenchaft. Wien 26, 1489-1514.

Seon, T., Hulin, J. P. \& Salin, D. 2004 Buoyant mixing of miscible fluids in tilted tubes. Phys. Fluids 16, L103.

Shin, J., Dalziel, S. \& Linden, P. 2004 Density currents produced by lock exchange. J. Fluid Mech. 521, 1-34.

Simpson, J. 1972 Effect of the lower boundary on the head of a gravity current. J. Fluid Mech. 53, $759-768$.

Simpson, J. 1997 Density Currents, 2nd edn. Cambridge University Press.

Stansby, P. K., Chegini, A. \& Barnes, T. C. D. 1998 The initial stages of dam-break flow. J. Fluid Mech. 374, 407-424.

StOKeR, J. J. 1957 Water Waves, Interscience.

UNGARISH, M. 2007 A shallow-water model for high-Reynolds-number gravity currents for a wide range of density differences and fractional depths. J. Fluid Mech. 579, 373-382.

Whitham, G. B. 1955 The effects of hydraulic resistance in the dam-break problem. Proc. R. Soc. Lond. A 227, 399-407.

Wilkinson, D. L. 1982 Motion of air cavities in long horizontal ducts. J. Fluid Mech. 118, 109-122.

ZALESAK, S. T. 1979 Fully multidimensional flux-corrected transport algorithms for fluids. J. Comput. Phys. 31, 335-362.

ZuKoski, E. E. 1966 Influence of viscosity, surface tension, and inclination angle on motion of long bubbles in closed tubes. J. Fluid Mech. 25, 821-840. 